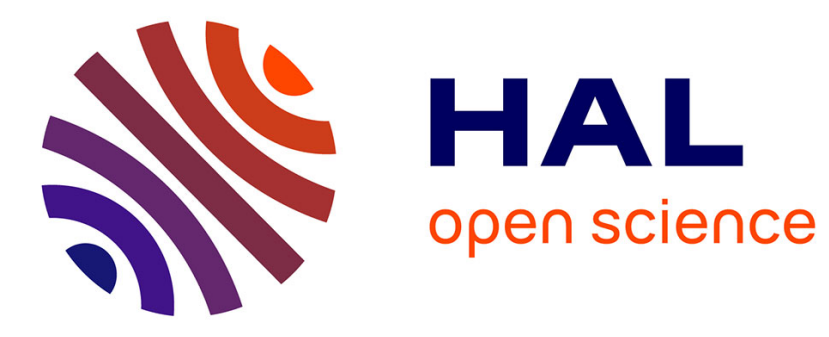

\title{
Le site gaulois
}

Philippe Barral, Gérard Bataille, Anne-Sophie Bride, Jean-Paul Guillaumet, Luc Jaccottey, Lucile Jeunot, Valérie Pichot, Martin Schönfelder

\section{To cite this version:}

Philippe Barral, Gérard Bataille, Anne-Sophie Bride, Jean-Paul Guillaumet, Luc Jaccottey, et al.. Le site gaulois. Gallia - Archéologie de la France antique, 2007, Epomanduodurum, une ville chez les Séquanes: bilan de quatre années de recherche à Mandeure et Mathay (Doubs), 64, pp.371-390. 10.3406/galia.2007.3319 . hal-01911682

\section{HAL Id: hal-01911682 \\ https://hal.science/hal-01911682}

Submitted on 7 Feb 2020

HAL is a multi-disciplinary open access archive for the deposit and dissemination of scientific research documents, whether they are published or not. The documents may come from teaching and research institutions in France or abroad, or from public or private research centers.
L'archive ouverte pluridisciplinaire HAL, est destinée au dépôt et à la diffusion de documents scientifiques de niveau recherche, publiés ou non, émanant des établissements d'enseignement et de recherche français ou étrangers, des laboratoires publics ou privés.

\section{(ㅇ)(1) $\$$}

Distributed under a Creative Commons Attribution - NonCommercial - NoDerivatives| 4.0 
hydrologiques dans la vallée du Doubs ouvre des perspectives très intéressantes pour les reconstructions paléoenvironnementales holocènes.

Ces investigations ont permis de mettre en évidence de nouvelles traces d'occupation protohistorique indiscutables dans cette partie du fond de la vallée du Doubs.

En ce qui concerne la taphonomie du site, on retiendra que les structures conservées dans les couches superficielles du lit majeur de la rivière le sont à l'état résiduel, ce qui rend a priori difficile, mais pas impossible, leur détection par les méthodes géophysiques de surface.

Dans le secteur du complexe monumental sanctuairethéâtre, survolé à maintes reprises à basse altitude, la reconnaissance géophysique a confirmé l'existence des structures archéologiques apparues fugitivement en prospection aérienne mais elle a révélé surtout d'autres structures dont l'existence était jusque-là insoupçonnée ou qui étaient matérialisées par de faibles concentrations de vestiges à la surface du sol.

Ces découvertes successives modifient radicalement la perception initiale que l'on avait de l'organisation spatiale des vestiges archéologiques dans notre zone d'étude. Elles posent la question de la compréhension de leur insertion dans la trame urbaine de l'agglomération à l'époque antique, à laquelle maintenant seule la fouille archéologique peut répondre en identifiant la nature et en précisant la fonction et la chronologie des structures détectées.

\section{LE SITE GAULOIS}

\section{INTRODUCTION}

La ville gallo-romaine a longtemps éclipsé le site gaulois, perçu essentiellement à travers des découvertes ponctuelles disparates et le lot d'objets trouvé au XIX ${ }^{\mathrm{e}}$ s. sous le grand temple romain, situé en vis-à-vis du théâtre. L'étude exhaustive de cet ensemble, qui n'avait jamais été réalisée, associée à celle d'autres vestiges plus modestes, conduit à réévaluer la nature et le statut de l'occupation gauloise. La réussite de la ville gallo-romaine s'explique certainement, au moins en partie, par la présence d'un centre économique et religieux déterminant un pôle de peuplement de première importance à la fin de l'âge du Fer. Mandeure fait en effet probablement partie, avec Verdun-sur-le-Doubs et Besançon, des trois principales agglomérations de plaine qui rythment le couloir de la moyenne vallée du Doubs, axe structurant du pays séquane (Barral, 2003).
La situation précise du site d'Epomanduodurum/Mandeure (fig. 5), un peu en retrait par rapport à l'axe est-ouest de la moyenne vallée du Doubs, peut-être considérée comme une anomalie, au regard des intérêts stratégiques romains, mais apparaît tout à fait logique dans le contexte territorial de l'âge du Fer. Le sanctuaire et l'agglomération gauloise d'Epomanduodurum se trouvent sur l'axe nord-sud de pénétration du massif du Jura par la haute vallée du Doubs, primordial pendant les âges des Métaux pour les échanges entre le plateau Suisse et le nord de l'Italie, d'une part, et le bassin rhénan, d'autre part (Millotte, Lambert, 1996 ; Barral et al., à paraître). Mandeure se situe par ailleurs en limite des territoires séquane et rauraque, position qui a sans doute une signification dans l'émergence d'un grand sanctuaire de territoire ${ }^{4}$.

Le site gaulois comprend trois composantes principales : dans la partie interne de la boucle du Doubs, et au nord-ouest, des témoins d'habitat (fig. 6, $\mathrm{n}^{\text {os }} 1-6$ ) ; au sudouest, en rive gauche, un espace funéraire (fig. $6, \mathrm{n}^{\circ} 11$ ) (Mougin et al., 1994 ; Barral, 1996) ; dans la partie sud-ouest du coude du Doubs, en rive droite, un sanctuaire, cour d'un complexe monumental au Haut-Empire (fig. 6, $\mathrm{n}^{\circ}$ 9). Une fréquentation du site dans les premiers temps du second âge du Fer est attestée par deux fibules de type Duchcov, trouvées anciennement. L'une provient du nord du site, Saint-Symphorien (fig. 6, no 1) (Tuefferd, 1878, pl. XVII), et une seconde est attribuée au sanctuaire (inédite, conservée au musée de Dijon : documentation Y. Jeannin). Une petite série d'objets du sanctuaire se rapporte au $\mathrm{III}^{\mathrm{e}} \mathrm{s}$. (La Tène C1), tandis que la phase qui est pour l'instant la mieux illustrée, dans le sanctuaire et la nécropole, correspond au $\mathrm{II}^{\mathrm{e}}$ s. (La Tène C2-D1).

P. B.

\section{L'HABITAT GAULOIS}

Les traces d'habitat sont, dans l'état actuel des connaissances, peu spectaculaires mais, réparties en différents points à l'intérieur du méandre du Doubs, elles constituent un ensemble topographiquement cohérent. Leur caractère discontinu est clairement lié aux aléas des recherches. Ces vestiges d'occupation précoce ont en effet été mis au jour à l'occasion de sondages ou de surveillances de travaux, dans des secteurs qui n'ont jamais fait l'objet de fouilles extensives. Entre 1959 et 1963, au lieu-dit Aux Arbues (fig. 6, $n^{\circ} 2$ ), sur un site de pied de coteau en bordure de la plaine

4. Sur le statut des Rauraques et leurs relations avec les Séquanes, voir Fichtl, 2000 et 2004, p. 104-107. 


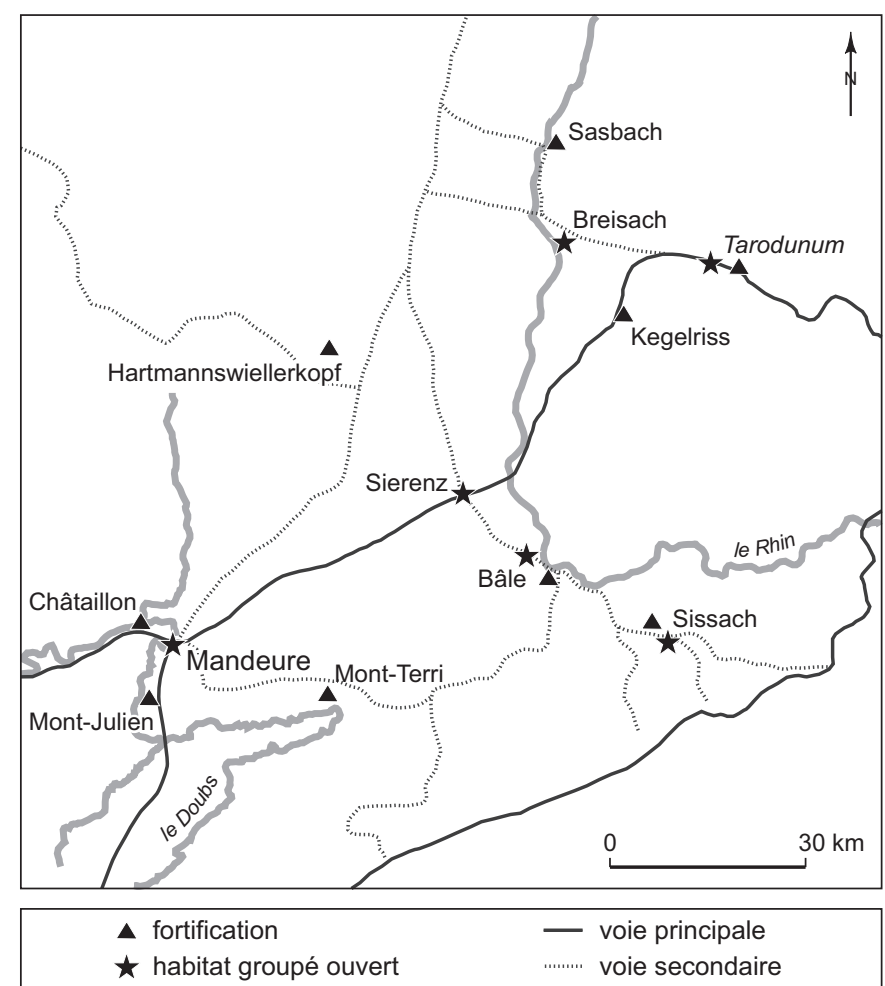

Fig. 5 - Mandeure et le territoire rauraque à l'époque gauloise (DAO : P. Barral, LCE, d’après Jud, Kaenel, 2002, complété).

alluviale du Doubs déjà occupé au Bronze final-Hallstatt C, des fouilles ont mis au jour de nombreux fragments d'amphores vinaires italiques Dressel 1, ainsi que deux fonds d'habitat semi-excavés de La Tène finale (Jeannin, 1986, p. 59 ; Jeannin, Laubenheimer, 1989, p. 75). Dans la boucle du Doubs, des travaux d'égouts effectués en 1968 rue des Bains (fig. $6, \mathrm{n}^{\circ} 6$ ) et des aménagements pour la construction d'un lotissement en 1974 au lieu-dit En Coudroie (fig. 6, no5) ont amené à la découverte de petits lots de matériels céramiques et amphoriques de la fin de l'époque gauloise (La Tène D-Auguste) (Jeannin, Laubenheimer, 1989, p. 75-77). Est à signaler la présence de fragments de vases balustres à pâte claire et de pots à cuire ou à conserver, dont les formes et les décors se retrouvent dans le mobilier de l'oppidum de Besançon (pour Besançon, voir Barral et al., 2005). Tout récemment, des sondages linéaires réalisés en trois endroits (fig. $6, \mathrm{n}^{\mathrm{os}} 4,7,8$ ) ont révélé l'existence de niveaux d'occupations précoces, livrant un matériel caractéristique de La Tène $\mathrm{D}$ et de l'époque augustéenne (inédit). Au vu de ces données, on peut avancer l'hypothèse que, pour l'essentiel, l'habitat gaulois se trouve enfoui sous la ville romaine, à l'intérieur de la boucle du Doubs et sur ses abords. Son extension et sa densité ne sont pas connues.
De plus, pour l'instant, les contextes d'occupation précoce semblent se rattacher exclusivement à la phase La Tène D2-Auguste, tandis que les contextes d'habitat plus anciens de La Tène C2-D1, contemporains du sanctuaire et de la nécropole, nous échappent encore. Par ailleurs, les prospections à grande échelle et sondages linéaires réalisés ces dernières années n'ont pas détecté la présence d'un rempart laténien. Nous excluons donc, pour l'instant, une configuration d'oppidum, au profit d'une agglomération de plaine ouverte, bénéficiant d'un réseau défensif de petits sites de hauteur fortifiés implantés le long des vallées du Doubs et de ses affluents (Barral, 2003).

P. B., L. Ja., V. P.

\section{LES MONNAIES GAULOISES PROVENANT DE MANDEURE}

Une conséquente série monétaire gauloise est issue du site de Mandeure-Mathay. Sur les quelque 346 monnaies recensées actuellement, 246 sont conservées au musée de Montbéliard et appartiennent au fonds ancien, attribué à Mandeure et étudiées une première fois il y a une quarantaine d'années (Colbert de Beaulieu, 1960). Sur cet ensemble, 87 sont identifiées comme provenant du sanctuaire par les registres d'entrée du musée (Capitole). Les 159 autres proviennent de Mandeure, sans plus de précision ${ }^{5}$. Une partie pourrait également être issue du sanctuaire, sans certitude. En ce qui concerne ce dernier, C. Duvernoy évoque la découverte de 324 monnaies gauloises, dont 44 en argent, chiffre qui ne tient évidemment pas compte des monnaies dispersées dans des collections particulières. On ne saurait également passer sous silence les huit lingots, vestiges du trésor du temple, trouvés dans une sorte de caveau à l'intérieur du sanctuaire, à la surface desquels on voyait encore le relief de quelques monnaies gauloises à légende QDoci Sam F (Jeannin, 1986, p. 54).

Une petite centaine de monnaies, découvertes en prospection, sont venues enrichir le corpus des monnaies manduriennes ces dernières années. Une quinzaine provient de différents endroits à Mandeure et Mathay, le lot principal ayant été trouvé en un seul lieu, sur une hauteur qui domine le site du sanctuaire du Clos du Château : le Châtelet (fig. 6, no 10).

$\mathrm{Au}$ total, nous disposons d'un échantillon représentatif de la circulation monétaire dans un secteur situé à l'interface des circulations est-ouest et nord-sud, qui draine donc

5. Colbert de Beaulieu mentionne 275 monnaies conservées à Montbéliard, 116 constituant le legs H. L'Épée et 159 la collection particulière R. Cuisenier. 


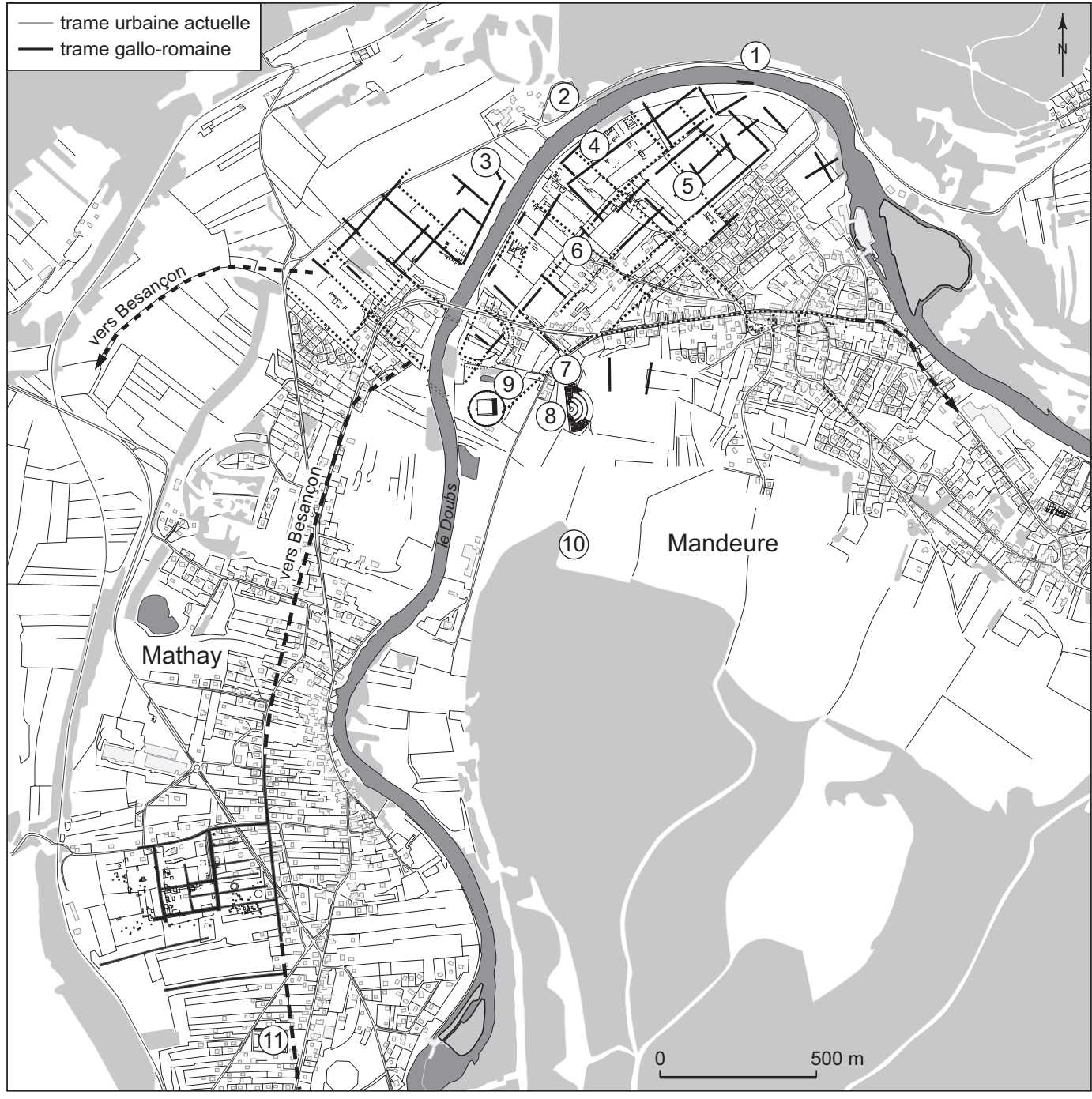

1. Saint-Symphorien, vers la fin du XIXe s. : découverte isolée, La Tène B1
2. Aux Arbues, 1959-1963: fonds d'habitat, amphores, fibule, La Tène D
3. Les Comboles, $2005:$ amphores, céramique, monnaie (prospection), La Tène D
4. Champs Pelletier, $2005:$ fosses, céramique, La Tène D-Auguste
5. En Coudroie, $1974:$ céramique, amphores, La Tène D-Auguste
6. Rue des Bains, $1968:$ céramique, amphores, La Tène D-Auguste
7. Sous la Grande Planche, $2005:$ céramique, fibules, La Tène D-Auguste
8. Les Ouchottes, $2002:$ fosses, céramique, La Tène C-D
9. Clos du Château, $1880:$ sanctuaire La Tène B-Auguste
10. Le Châtelet, vers $1990-2000:$ monnaies et objets divers (prospection), La Tène D
11. Les Longues Raies, 1993-1995: nécropole, La Tène C2-D1

Fig. 6 - Répartition des lieux de découverte des vestiges de la fin de l'âge du Fer à Mandeure (données et DAO : P. Barral, L. Jaccottey, LCE; fond de plan D. Watts et P. Mougin, SIVAMM, modifié par P. Barral).

tout particulièrement les numéraires local, régional et interrégional. Il convient cependant de garder à l'esprit le fait qu'une majorité des monnaies de Mandeure-Mathay, disponibles à ce jour, provient du sanctuaire de Mandeure et d'un deuxième site proche, probablement cultuel également, et que leur mode de déposition et de rassemblement obéit à des règles particulières.

Nous considérons ci-dessous, de façon globale, l'ensemble des monnaies répertoriées sur l'agglomération. Les monnaies gauloises de Mandeure se répartissent inégalement 
en quatre groupes: les monnaies en or (moins de $1 \%$ ), en argent $(17 \%)$, en alliage cuivreux (11\%) et en potin $(71,5 \%)$. Les monnaies des trois premiers groupes sont frappées, celles du dernier groupe, coulées.

Les deux monnaies d'or ont été trouvées en 1903 en rive gauche du Doubs, au lieu-dit Romont, à peu de distance de la nécropole gauloise de Mathay (Parent, 1965 ; Dayet, 1967) (fig. 6, no 11). Il s'agit d'un statère au triskèle (LT 8897) et d'un statère à la roue (LT 8901), tous deux traditionnellement attribués aux Séquanes.

Les monnaies d'argent les mieux représentées à Mandeure (et les plus répandues de façon générale en Gaule de l'Est) sont des types interrégionaux qui s'inspirent de deniers de la République romaine, dits à la Rome casquée (fig. 7). Le demi-denier gaulois ou quinaire dit de Kaletedoy (LT 8178-8291) présente à l'avers une tête casquée avec une légende abrégée KAL et au revers un cheval harnaché. Il est très répandu dans l'est de la France et plus particulièrement chez les Éduens, les Séquanes et les Lingons, qui l'auraient fabriqué conjointement, en nombreuses déclinaisons locales ou régionales. On peut ainsi évoquer une zone économique monétaire unifiée, expression d'une volonté politique, dont les bases ont été posées dans les dernières décennies du $\mathrm{II}^{\mathrm{e}}$ s. av. J.-C. (Colbert de Beaulieu, 1965 et 1973 ; Fischer, 1999 ; Gruel, Morin, 1999). On recense 16 exemplaires de ce type à Mandeure, ce qui est peu, surtout si l'on considère la proximité de lieux de production. Une fabrication du denier de Kaletedoy est en effet attestée au Mont Vully (Auberson, Geiser, 2001) et probablement à Bâle, chez les Rauraques. Quelques autres types viennent toutefois doubler le corpus des quinaires d'argent traditionnellement attribués aux Séquanes (LT 5405-5411: 9 ex. ; LT 5550 : 6 ex. ; LT 5639 : 1 ex.).

Les autres types de monnaies d'argent sont présentes sur le site en un, deux ou trois exemplaires, très rarement plus, et proviennent de différentes régions, parfois éloignées. On recense ainsi quelques monnaies des voisins Éduens (LT 5138-5252: 5 ex. ; LT $4871: 1$ ex.), du nord de la Suisse (LT 9322: 2 ex.), du sud de l'Allemagne (LT 2172-2176: 1 ex.), quelques-unes des peuples de Gaule de l'Ouest (Pictons : LT 4484 ; Bituriges Cubes : LT 4097 et LT 4139 4142), quelques autres des populations de la basse vallée du Rhône (Marseille: LT 689 ; vallée du Rhône: LT 5762 et LT 5777-5779 ; Cavares : LT 2621).

Le numéraire le plus répandu, à Mandeure comme de façon générale sur les sites de la fin de l'époque gauloise, est le potin qui désigne une monnaie coulée, en alliage de cuivre, étain, plomb et antimoine, fabriquée en série par la technique du moulage en grappe. Leur production massive va de pair avec une multiplication des lieux de fabrication et des types iconographiques. Certains grands types sont présents en nombre sur les territoires de plusieurs peuples (potin à la grosse tête: Gruel, Geiser, 1995; potin au sanglier : Fichtl, 2004, p. 83-84). On observe également que ces grands types se déclinent en variantes chronologiques et/ou microrégionales (Gruel dir., 1995).

Le numéraire associant deniers d'argent et potins, à légende Togirix, est traditionnellement attribué aux Séquanes, en fonction de leur aire de répartition. La série des potins, présents en très grand nombre à Mandeure (124 ex.), figure au droit une tête casquée et au revers un lion bondissant (fig. 7). Elle appartient aux émissions de potins tardives, comme l'indique la présence d'une légende épigraphique (le nom de Togirix apparaît au revers, soit sous sa forme abrégée, TOC, soit sous une forme plus complète TOGIR). Quatre variantes (présentes dans le lot provenant de Mandeure), ont été discernées d'après le dessin du droit. Elles traduisent une romanisation progressive du motif de la tête casquée, ellemême reflétant probablement l'évolution sociale du personnage. Togirix serait un notable séquane qui aurait commandé un corps de troupes auxiliaires, sous les ordres de César, pendant la guerre des Gaules (Colbert de Beaulieu, 1962 ; Dayet, 1962). Un autre de ces chefs gaulois, probablement séquane, récompensé par l'attribution des tria nomina romains, est illustré par le numéraire associant monnaies d'argent et potins, à légende QDoci Sam F (Dayet, 1964). D’autres types considérés comme séquanes (LT 5611, 5527, 5508, 7011) sont attestés par un petit nombre d'exemplaires à chaque fois.

Le type de potin qui arrive en seconde place à Mandeure (63 exemplaires, soit $18 \%$ du total) est le type dit «à la grosse tête " LT 5368, très répandu de la Bourgogne à la Suisse. Une partie du numéraire est vraisemblablement séquane, mais ce peuple ne détient certes pas le monopole de la fabrication de cette série, si l'on en juge par son aire de diffusion principale. La répartition par classes de ce numéraire (Geiser, Gruel, 1992; Gruel, Geiser, 1995) montre la forte proportion à Mandeure des potins à la grosse tête à bandeau lisse (GT A), au détriment des séries à bandeau décoré (GT B), plus tardives, figurées ici seulement par quatre monnaies (fig. 7). Les variantes les mieux représentées numériquement sont les classes GT A3, GT A4.1 et GT A6.1. Il s'agit de classes que nous rencontrons principalement dans l'est de la France ainsi qu'en Suisse. Les variantes présentes à Mandeure semblent appartenir aux émissions les plus précoces, de La Tène D1 (Gruel, Geiser, 1995). 

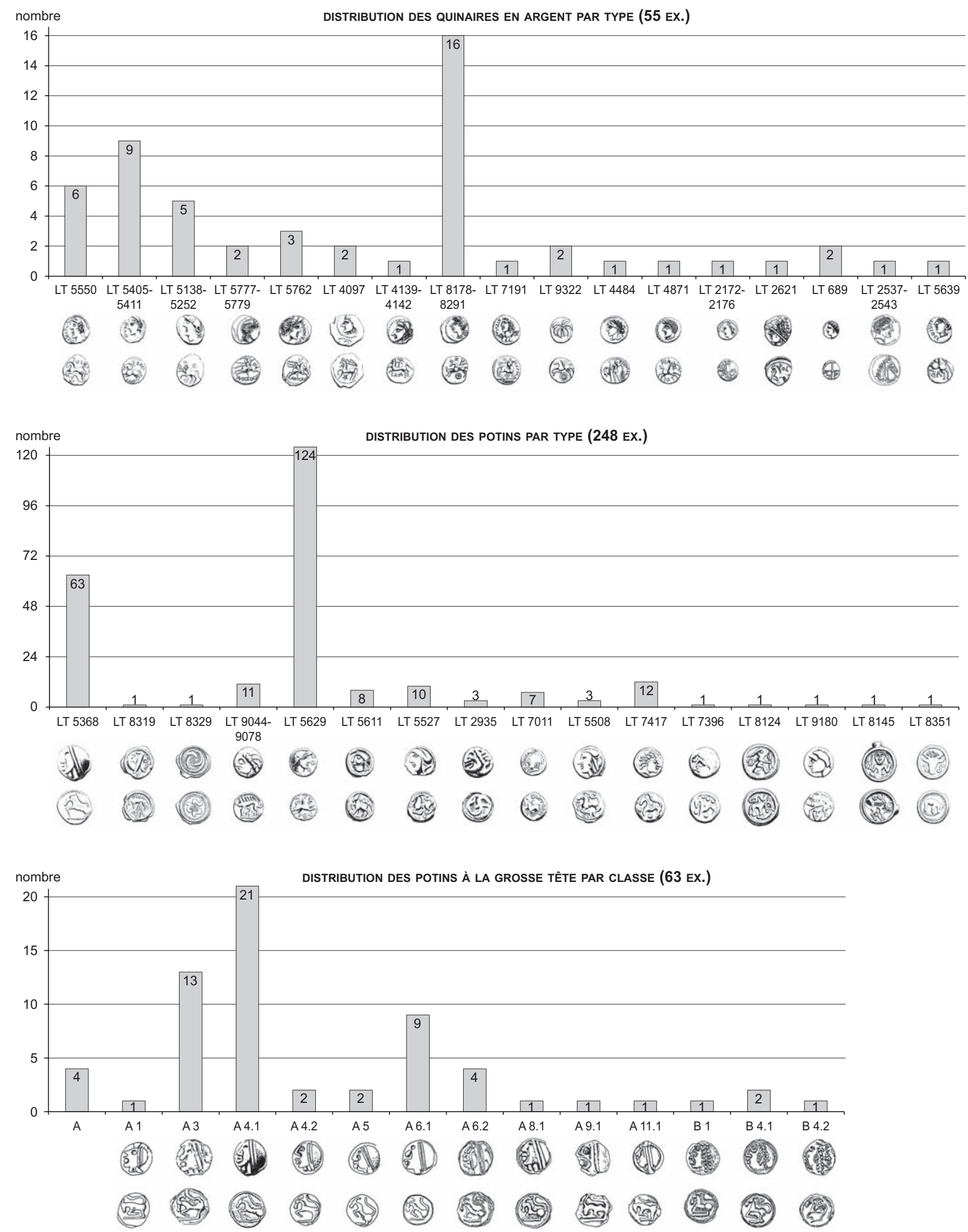

Fig. 7 - Histogrammes de distribution par type ou classe des quinaires et des potins de Mandeure (données et DAO : L. Jeunot). 
Les autres types de potins, de régions proches ou éloignées, sont attestés en quelques exemplaires. Ceux qui sont les mieux représentés sont le potin au sanglier attribué aux Leuques et Médiomatriques (LT 9044-9078 : 11 ex.) et le potin au cheval identifié aux Sénons (LT 7417 : 12 ex.). Le nord de la Gaule est documenté par plusieurs types qui figurent à chaque fois en un seul exemplaire (Rèmes : LT 8124, LT 8145, LT 8351 ; Parisii, Bellovaques ou Meldes : LT 9180). On est frappé en comparaison par la faible proportion de certains types de potins des peuples voisins (potin éduen LT 2935 illustré en trois exemplaires ; potins lingons LT 8319 et LT 8329, attestés en un seul exemplaire).

Le numéraire de bronze frappé réunit 30 monnaies qui se répartissent inégalement entre deux types considérés comme séquanes (LT 7005 : 10 ex. ; LT 5594-5604 : 14 ex.), probablement assez tardifs, et différents types attestés en un ou deux exemplaires, attribués aux peuples du nord de la Gaule (Rèmes : LT 8040 ; Veliocasses : LT 7333) et du sud de la Gaule (région de Marseille : LT 1673, LT 2227 ; Volques Arécomiques : LT 2677) (fig. 8).

$\mathrm{Au}$ sein de cet ensemble de 346 monnaies provenant de Mandeure, les espèces de faible valeur forment le lot principal (potins et bronzes frappés : 80,5\%; statères en or et quinaires en argent: 16,5\%; indéterminées : $3 \%$ ). Le monnayage local ou régional (pays séquane) l'emporte très nettement (fig. 9). Le chiffre de $56 \%$ doit être considéré comme un minimum, puisqu'il faut l'augmenter d'une partie, difficile à estimer, des potins à la grosse tête. Les séries attribuables aux peuples voisins (Éduens, Lingons, Leuques-Médiomatriques...) sont peu présentes et les régions éloignées ne sont illustrées que par quelques monnaies. Au total, le monnayage ne semble pas refléter de façon très évidente l'insertion du pays mandurien (et audelà du territoire séquane) dans la vaste zone d'échanges dynamiques de la fin de l'âge du Fer.

D'un point de vue chronologique, en l'absence de données contextuelles, on ne peut se référer qu'à des indications typochronologiques. Un lot très majoritaire de monnaies de Mandeure correspond à un faciès de circulation tardif, attribuable au milieu et à la seconde moitié du $\mathrm{I}^{\mathrm{er}} \mathrm{s}$. av. J.-C. Certains types de potins à la grosse tête, présents en nombreux exemplaires, notamment dans le sanctuaire, sont en revanche précoces, attestés dès La Tène D1. L'abondance de monnaies tardives de type Togirix pourrait être liée à la romanisation du site et suggérer même une présence militaire marquée, dans la seconde moitié du I ${ }^{\mathrm{er}} \mathrm{s}$. av. J.-C.

L. Je.

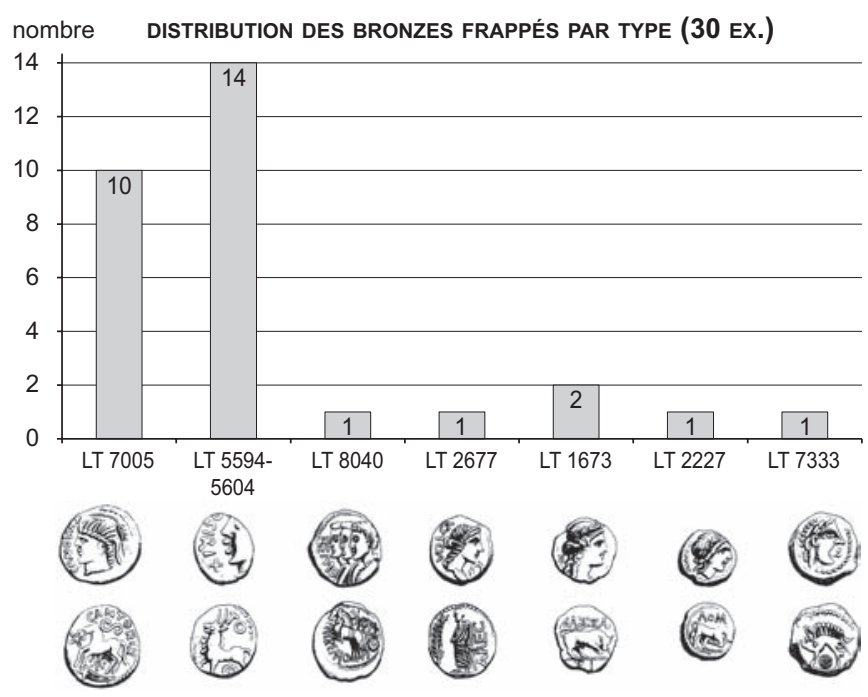

Fig. 8 - Histogramme de distribution par type des bronzes frappés de Mandeure (données et DAO: L. Jeunot).

\section{LE SANGTUAIRE GAULOIS}

Il est localisé, tout ou en partie, sous le grand temple romain à péribole circulaire qui fait face au théâtre. Son emprise, son organisation ne sont pas connues. On peut, du moins, cerner quelques-unes de ses caractéristiques majeures à travers le mobilier qui nous est parvenu.

\section{CONTEXTE DES DÉCOUVERTES, NATURE ET CHRONOLOGIE DU MOBILIER}

L'hypothèse de l'existence d'un sanctuaire gaulois antérieur à l'édifice identifié comme temple gallo-romain (voir infra, p. 390 sq.), situé en vis-à-vis du théâtre près de la rive droite du Doubs, a été émise par plusieurs auteurs, en raison de la présence de nombreux objets de la période gauloise et notamment de deux lots importants de monnaies, d'une part, de bracelets et anneaux en verre coloré, d'autre part. L'extrême dispersion du matériel entre les musées a été jusqu'à ces dernières années un frein à l'étude de cet ensemble, emblématique des offrandes déposées dans les grands sanctuaires celtiques.

On dispose de données très succinctes sur les vestiges enfouis sous le supposé temple romain, ce qui est dû sans doute à la fois aux conditions de l'exploration et au caractère ténu, peu spectaculaire, des structures du sanctuaire laténien. Est à retenir la mention par C. Duvernoy (1883b) de l'existence de "très anciens murs en pierres sèches de grandes dimensions ", à l'intérieur de l'enceinte du péribole, et la découverte de nombreuses monnaies 


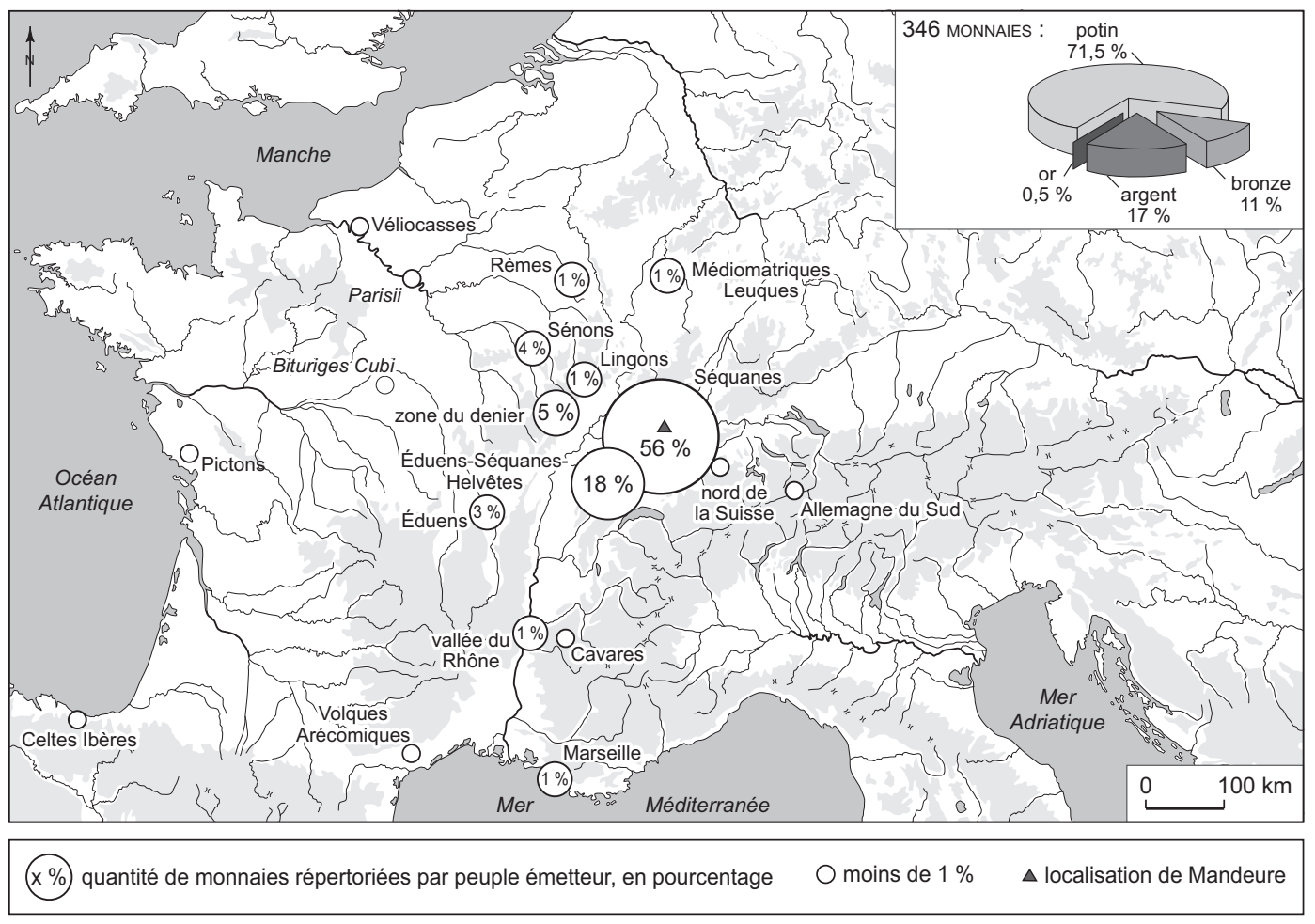

Fig. 9 - Carte de provenance des monnaies de Mandeure et graphique de répartition par métal (données et DAO : L. Jeunot; fond de carte: B. Turina).

gauloises, entre $0,80 \mathrm{~m}$ et $1,20 \mathrm{~m}$ de profondeur, dans la zone comprise entre le perron du temple et l'entrée est du péribole (fig. 3).

Une indication importante concerne le contexte de découverte du lot mobilier dont la plus grande partie se trouve au musée de Saint-Germain-en-Laye. Elle est fournie par une lettre de l'inventeur, A. Péquignet, datée du $1^{\text {er }}$ avril 1909 (Barral et al., 2005, p. 40-41). Il y explique, entre autres, que l'intégralité du lot qu'il souhaite vendre au musée des Antiquités nationales provient d'une fosse d'à peu près $10 \mathrm{~m}^{2}$, située sous le bâtiment rectangulaire. Cette fosse était scellée par une couche de graviers de $80 \mathrm{~cm}$ d'épaisseur encore en place et sur laquelle avaient été trouvées de nombreuses pièces de monnaies du $\mathrm{I}^{\mathrm{er}} \mathrm{s}$. av. J.-C. Bien que succinctes, ces indications fournissent une ébauche de stratigraphie et de chronologie du sanctuaire gaulois et, surtout, attestent que l'on a affaire à un ensemble cohérent provenant apparemment d'une seule structure.

L'étude systématique des trouvailles du sanctuaire gaulois entreprise depuis quelques années (en premier lieu : Pichot, 1996) apporte des précisions sur la chronologie du sanctuaire gaulois. Trois étapes de fonctionnement peuvent être restituées. La phase initiale (La Tène B2-C1) est seulement illustrée par une petite série d'objets: fibules (non illustrées), attache zoomorphe de ceinture féminine, bracelet (fig. 10, $\mathrm{n}^{\text {os }} 1$ et 2 ). Une seconde phase (La Tène C2-D1) est attestée essentiellement par un gros dépôt en fosse (découverte Péquignet), qui forme un assemblage composite, au sein duquel dominent perles et bracelets en verre, mais qui comprend également d'assez nombreux anneaux (fig. 10, $\left.\mathrm{n}^{\text {os }} 10-15\right)$ et des fibules apparentées au type de Nauheim (fig. 10, $\mathrm{n}^{\text {os }}$ 5-9). Une troisième phase, tardive (La Tène D2Auguste), correspond à une abondante série de monnaies, à laquelle s'ajoutent des rouelles, anneaux bouletés, cages à grelots, etc. (fig. 10, $\mathrm{n}^{\mathrm{os}} 16-20$ ).

Les objets qui nous sont parvenus se répartissent entre différentes catégories fonctionnelles inégalement représentées: parure, vaisselle céramique, armement et harnachement, outillage, monnaies, amulettes, etc. (fig. 10 à fig. 14 et pl. XV, hors texte). Certains types d'objets figurent sous la forme de séries nombreuses (fibules, anneaux en bronze, bracelets et perles en verre, monnaies), d'autres en plusieurs exemplaires (vases céramiques miniatures:

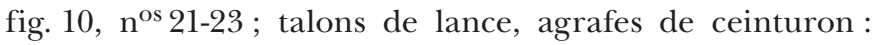
fig. 14 ; pièces décoratives de joug : fig. $10, \mathrm{n}^{\text {os }} 3-4$; miroirs 


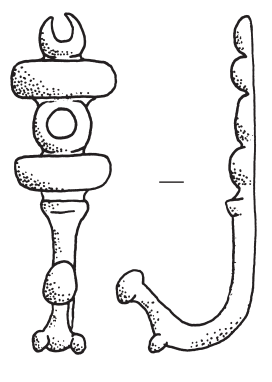

1

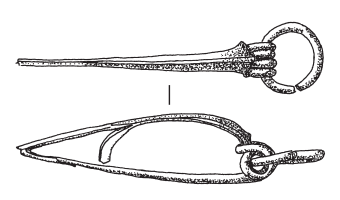

5
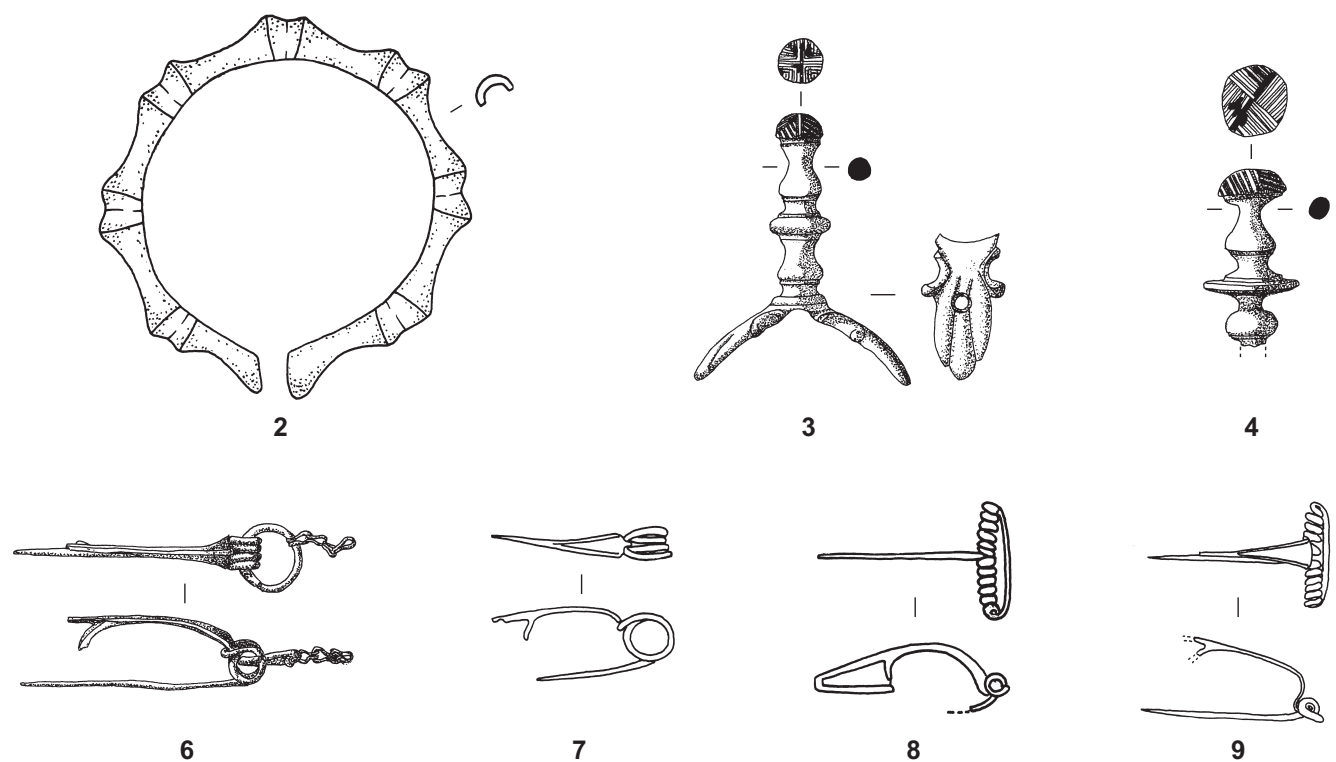

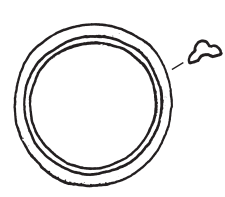

10

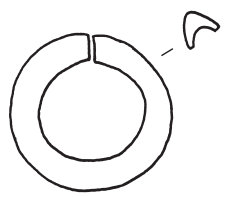

11

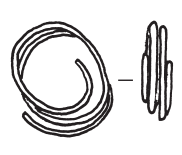

12

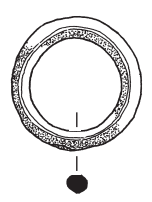

13

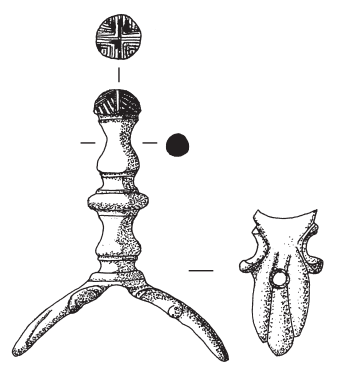

3

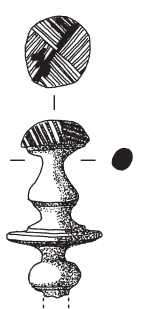

4

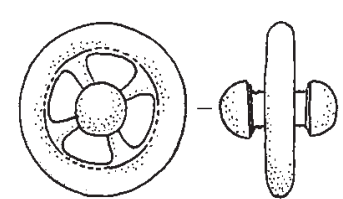

16

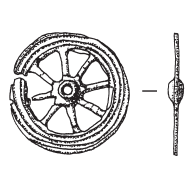

17

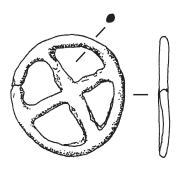

18

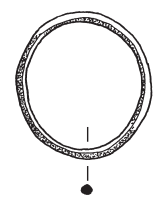

14

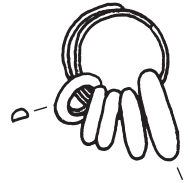

15
D

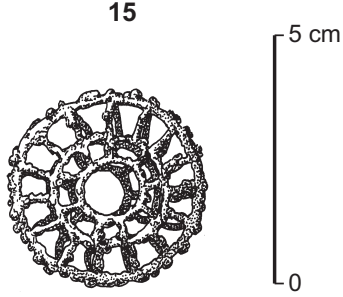

20

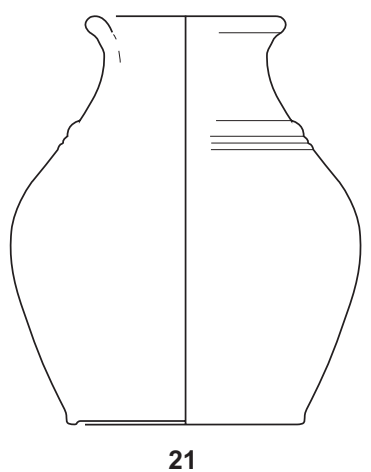

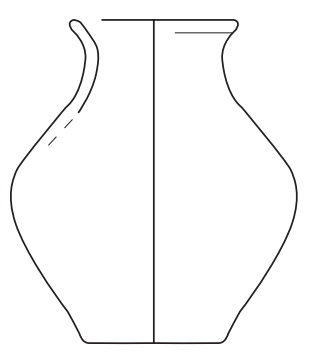

22

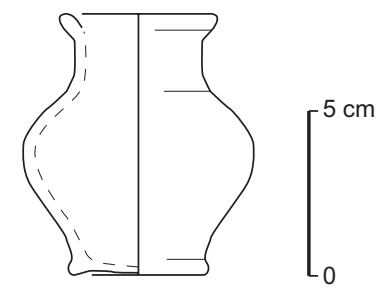

23

Fig. 10 - Le Clos du Château à Mandeure. Sélection de mobilier provenant du sanctuaire gaulois et conservé aux musées de Montbéliard et de Saint-Germain-en-Laye : 1, extrémité zoomorphe de chaîne de ceinture ; 2, bracelet (La Tène B2-C1) ; 3-4, pièces décoratives de joug ; 5-9, fibules apparentées au type de Nauheim (La Tène C2-D1) ; 10-15, anneaux (La Tène C2-D1) ; 16-20, rouelles, anneau bouleté et cage à grelots (La Tène D2-Auguste) ; 21-23, vases céramiques miniatures. Les pièces 1 à 20 sont en métal cuivreux à l'exception de 3 et 4, en métal cuivreux et verre opaque rouge, 5 et 6 en argent et 15 en métal cuivreux et verre (dessins : C. Mauduit et V. Pichot; DAO : P. Barral, LCE). 
en bronze étamé, éléments foliacés, carnyx : fig. 15 à fig. 18), d'autres enfin en exemplaires uniques (hache à douille en fer, poucier de passoire, etc.). De façon également notable cohabitent des objets intacts et des pièces tordues, lacérées, fragmentées. Des objets fabriqués en série, de faible valeur intrinsèque, voisinent avec des pièces relevant d'un haut savoir-faire technique.

Nous examinons plus précisément ci-dessous quelquesunes des catégories de mobilier représentées dans le sanctuaire de Mandeure.

$$
\text { P. B., L. Ja., V. P. }
$$

\section{LA CÉRAMIQUE}

C. Duvernoy signale que très peu de poterie a été trouvée dans les fouilles des années 1880: «quelques grossiers tessons à pâte quartzeuse ». Un groupe de cinq vases de taille miniature figure dans le lot vendu par A. Péquignet au musée de Saint-Germain-en-Laye (fig. 10, $\mathrm{n}^{\text {os } 21-23) .}$ Il s'agit de petits pots ovoïdes tournés, dont l'un présente une surface claire, les quatre autres des surfaces grises ou noires, lissées. Ces vases de petite taille tournés sont caractéristiques des dépôts de sanctuaires et de nécropoles de la fin de l'âge du Fer. À Mirebeau-sur-Bèze (Côte-d'Or), des pots tout à fait identiques à ceux de Mandeure figurent en nombreux exemplaires dans deux dépôts en fosses du temple gaulois (Barral, Guillaumet, 1994). Ces dépôts sont datés de la seconde moitié du $\mathrm{II}^{\mathrm{e}} \mathrm{s}$. av. J.-C.

P. B.

\section{LE DÉPÔT DE BRACELETS ET ANNEAUX EN VERRE}

En 1909, au Clos du Château, A. Péquignet découvrit « dans un trou de 2 mètres de profondeur sur huit à dix $\mathrm{m}^{2}$ [...] sous une couche de gravier de $80 \mathrm{~cm}$ d'épaisseur » 377 «anneaux» en verre, auxquels étaient associés différents objets, ainsi que des monnaies. Le musée des Antiquités nationales se porta acquéreur de cet ensemble la même année ${ }^{6}$. Plusieurs spécimens ont également été acquis à la

6. Après une âpre négociation dont témoigne toute une série de lettres conservées dans les archives du musée des Antiquités nationales à Saint-Germain-en-Laye. L'achat fut enregistré sous le numéro global 52 491. Le musée possède à l'heure actuelle 406 pièces identifiées comme provenant du dépôt, ce qui fait une différence de 29 objets par rapport à l'ensemble de départ. D'autres achats furent effectués par le musée, toujours au profit de A. Péquignet et concernent, semble-t-il, des objets gallo-romains. Une donation fut faite par S. Reinach en 1914 de plusieurs pièces «acquises sur les lieux » (Guillard, 1989a, p. 24). Les exemplaires en verre, surnuméraires par rapport au lot initial, peuvent probablement être associés à ces achats et dons, entre même période par les musées de Besançon, Montbéliard et Belfort, tous identifiés comme provenant du sanctuaire (Pichot, 1996; Bride, 1999). Il s'agit selon toute vraisemblance d'exemplaires provenant de la découverte Péquignet (pl. XV, hors texte).

L'ensemble conservé à Saint-Germain n'a été étudié pour la première fois qu'en 1989 (Guillard, 1989a et b). Une nouvelle étude, portant sur l'ensemble des pièces conservées dans les différents musées, a été réalisée par nos soins en 2001 (Bride in Barral dir., 2001). Nous en avons tiré les informations essentielles permettant de caractériser ce dépôt d'objets en verre.

Le corpus disponible à ce jour réunit 541 pièces se répartissant en 163 bracelets, 368 perles, 9 probables têtes d'épingles et un fragment de vase à parfum. Il s'agit d'un corpus typologiquement et chronologiquement cohérent, si l'on excepte la présence de 4 ou 5 pièces, certainement gallo-romaines d'après leur typologie et leur couleur, dont le caractère intrusif est probable ${ }^{7}$.

Les objets sont, pour la plupart, en parfait état de conservation et présentent peu, voire pas, de trace d'usure évidente due à une utilisation normale. Tout au plus peuton relever des attaques naturelles de corrosion. Ce rassemblement est dominé par deux groupes principaux : bracelets et anneaux. On notera que la majorité des bracelets figure sous une forme fragmentaire (près de $80 \%$ ), le phénomène inverse caractérisant les perles (85\% intactes), sans que l'on connaisse la part du bris volontaire rituel, celle du bris accidentel (les perles sont moins fragiles que les bracelets), celle des cassures et manques liés aux conditions de la découverte. Le musée de Saint-Germain conserve par ailleurs plusieurs parures annulaires composées d'un bracelet filiforme en bronze à extrémités se chevauchant, sur lequel est accrochée une série d'anneaux en verre de couleur. L'hypothèse qu'il s'agisse de montages factices, postérieurs à la découverte, a été parfois émise, sans preuve.

\section{Les bracelets}

Les bracelets se répartissent en deux groupes principaux, l'un correspondant à des types larges côtelés, l'autre à des types filiformes (fig. 11). Les bracelets moulurés laténiens (21 exemplaires) possèdent généralement cinq

1909 et 1914. Leur identification à l'intérieur du lot conservé au musée est impossible.

7. Il n'est pas certain qu'ils aient fait partie de la trouvaille principale (voir note 6). L'occupation du site au Haut- et au Bas-Empire, bien attestée, suffit à expliquer leur présence. 


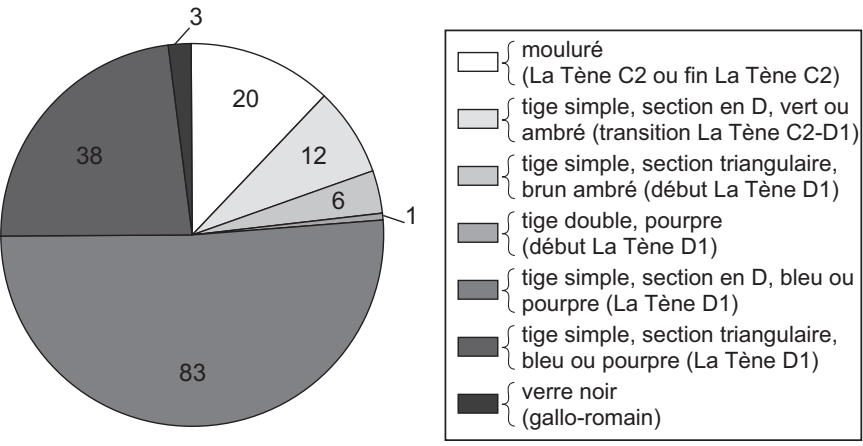

Fig. 11 - Répartition typochronologique des bracelets (données et DAO : A.-S. Bride).

moulures et la nervure centrale présente fréquemment un décor plastique (perles, tresse). La majorité est en verre incolore à couverte interne jaune opaque, quelques-uns sont en verre bleu cobalt, brun miellé ou vert. La combinaison bracelet mouluré-verre brun miellé est peu fréquente et n'apparaît d'ailleurs pas dans la typologie actuellement en vigueur (Gebhard, 1989b).

Les bracelets filiformes à tige simple se composent de deux groupes typologiques inégaux, en fonction de la forme de leur section. Les bracelets à tige simple et section en D sont les plus largement représentés (95 ex.). Soixanteneuf sont en verre pourpre, quatorze en verre bleu cobalt, douze en verre brun miellé ou vert. La grande majorité sont lisses, mais une dizaine, en verre bleu, violet, brun miellé ou vert, présentent un décor rapporté sous forme d'un filet zigzaguant en verre opaque jaune ou blanc. Un exemplaire en verre de base brun miellé, conservé au musée de Montbéliard, est pourvu d'un décor lacunaire de minces fils de verre jaune opaque. Il s'agit d'une nouvelle variante d'un type rare attesté, en verre translucide incolore à fils jaunes, à Mirebeau-sur-Bèze en Côte-d'Or, et en verre bleu à fils jaunes à Besançon dans le Doubs (Guilhot, Goy dir., 1992 ; Bride, 1999). Un autre exemplaire de ce type a été identifié récemment sur le site de Bibracte dans la Nièvre (Bride, 2006).

Les bracelets à tige simple et section triangulaire (44 ex.) sont en verre bleu ou pourpre (respectivement 11 et 27 pièces), plus rarement brun miellé (6 ex.).

Quatre pièces se distinguent clairement au sein du corpus des bracelets : deux exemplaires à épaisses moulures transversales, deux exemplaires massifs à section ronde, l'un à surface striée et tige ouverte. Par leur facture et leur couleur de base (noir à olive sombre), qui imite le jais (le verre noir tend à remplacer cette matière au Bas-Empire), ils sont attribuables à l'époque gallo-romaine.
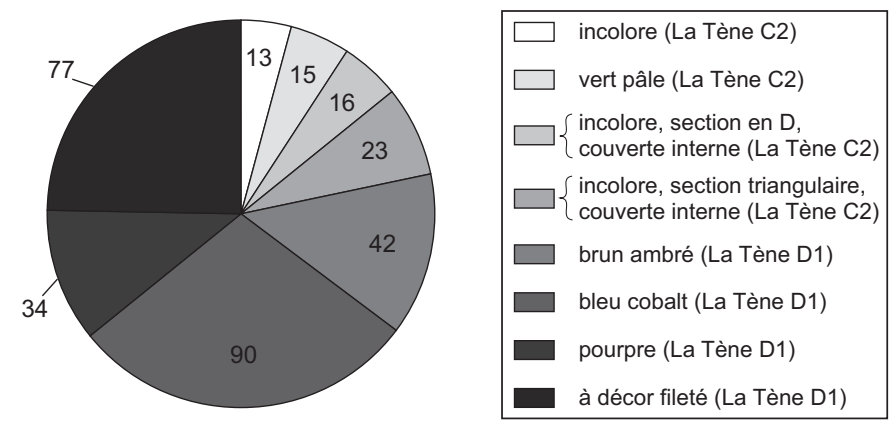

Fig. 12 - Répartition typochronologique des perles (données et DAO : A.-S. Bride).

\section{Les perles}

Les perles sont majoritaires au sein du corpus (368 ex.). Elles présentent une large gamme de décors et de couleurs (fig. 12). Leur fabrication semble parfois moins soignée que celle des bracelets (verre de base pâteux, filandreux, décors maladroits). S'agit-il d'une production spécifique destinée à ce seul cadre religieux?

Les perles annulaires grêles monochromes prédominent. Elles sont réalisées en verre bleu cobalt (groupe VI de R. Gebhard : 90 ex.), pourpre (groupe X : 34 ex.), vert pâle (groupe IV : 15 ex.), brun ambré (groupe VIII : 42 ex.) ou incolore (groupe III : 13 ex.). Les perles massives à section en $\mathrm{D}$ sont à couverte interne jaune opaque (groupe I : 16 ex.), de même que les perles à section triangulaire (groupe If : 23 ex.). Les perles annulaires, plus ou moins massives, à décors de fils rapportés radiants (A) et spiraliformes (B), concentriques (C), mouchetés (D), de damiers (E) ou de zigzags (F) présentent une grande variété de combinaisons et totalisent 77 exemplaires. On ne recense qu'une seule perle à décor moucheté et six perles à décors de damiers, dans un mauvais état de conservation.

Une perle en verre noir à décor plastique peut être attribuée à la période gallo-romaine par son verre de base.

\section{Les têtes d'épingles}

Neufs pièces du musée des Antiquités nationales peuvent être identifiées comme des perles sur tige de fer, ou des têtes d'épingle. De couleurs et de tailles diverses, elles présentent des traces plus ou moins nettes de fer en leur centre et à leur base. Ce type d'objet est illustré sur le site de Wederath (Allemagne), comme l'atteste la tombe 1673 de ce site, datable de La Tène C2-D en raison de la présence de perles massives à section triangulaire et couverte interne, et d'une perle annulaire à décor fileté ondoyant (Cordie-Hackenberg, 
Haffner, 1991, pl. 435). N. Venclovà mentionne également la présence de ce type en Bohême, sur les sites de Stradonice et de Hrazany (Venclovà, 1990, pl. $44 \mathrm{n}^{\text {os }} 17$ et 18). Un autre exemplaire est attesté sur le site de Bibracte (Bride, 2006). D'une couleur vert sombre, décorée d'un fil jaune opaque, elle présente une trace d'arrachement à sa base et semble destinée à être regardée par le haut. Une identification comme tête d'épingle peut donc être envisagée pour ces objets. Leur datation doit être mise en relation avec celle des perles annulaires, en raison de la couleur de leurs verres de base et de leurs décors. On peut donc les placer à La Tène C2-D pour celles à décor monochrome et à La Tène D pour celles à décor fileté.

\section{Le vase à parfum}

Un fragment de verre bleu cobalt, creux, comportant à sa base trois petites boules de verre, est identifiable comme un petit vase à parfum réalisé sur noyau. L'intérieur de la pièce est rugueux et conserve des traces de sable.

\section{Conclusion}

Des comparaisons régionales peuvent être esquissées, en ce qui concerne certains types et associations présents dans ce corpus. Le site de Gasfabrik à Bâle a livré des bracelets lisses à section triangulaire en verre brun et des perles annulaires à décor ondoyant, types peu répandus dans le monde celtique (Furger-Gunti, Berger, 1980). Des similitudes existent également avec le mobilier de Besançon (fouilles du Parking de la Mairie: Guilhot, Goy dir., 1992 ; Bride, 1999), qui inclut plusieurs fragments de bracelets à section triangulaire en verre brun, à section en $\mathrm{D}$ verre pourpre et décor de filet jaune opaque, de perles à décor de damier. Quelques parallèles intéressants existent également, notamment pour des variantes de bracelets peu fréquentes, en verre brun ou vert, sur les sites de Verdun-sur-le-Doubs et Mirebeau-sur-Bèze (Bride, 1998 et 1999). Mentionnons enfin, plus éloigné, l'oppidum de Bibracte qui a livré une tête d'épingle ou perle sur tige de fer, un bracelet en verre brun à fils jaunes, des perles annulaires en verre incolore à couverte jaune ou à décor de damier (Bride, 2006).

La chronologie du dépôt s'étend de La Tène C2 à La Tène D1, voire jusqu'aux débuts de La Tène D2, si l'on considère la prédominance des perles à décors filetés, type de parure qui perdure au détriment des bracelets sur de nombreux sites au cours de la dernière étape laténienne (Feugère, 1992)

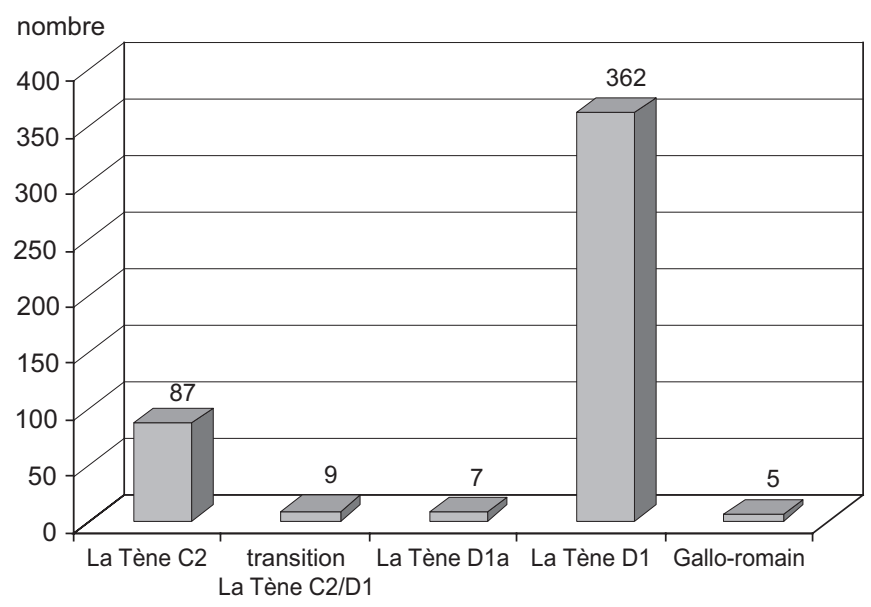

Fig. 13 - Distribution typochronologique de l'ensemble du mobilier en verre (données et DAO : A.-S. Bride).

(fig. 13). Les bracelets moulurés en verre bleu cobalt ou incolores à couverte interne, de même que les perles annulaires massives, à section triangulaire ou en D et couverte interne, ou les perles annulaires grêles monochromes, peuvent être attribués à La Tène C2 (Stähli, 1977 ; Gebhard, 1989a; Kaenel, 1990). Certains bracelets moulurés réalisés en verre brun miellé, absents de la typologie de Gebhard, peuvent être attribués avec précaution à la fin de La Tène C2 ou au tout début de La Tène D1, en raison de la couleur de leur verre de base. D'autres pièces, telles que les bracelets à tige simple, à section $\mathrm{D}$, réalisés en verre brun, ou en vert bouteille, marquent la transition La Tène C2-D1 (Gebhard, 1989a). De tels objets ont été mis au jour récemment dans des contextes bien datés fin La Tène C2-début La Tène D1, notamment à Verdun-sur-le-Doubs en Saône-et-Loire (Bride, 1999 ; Barral dir., 2001). Les perles annulaires monochromes bleu cobalt ou vert pâle posent quelques problèmes de datation, hors contexte. Elles apparaissent en effet à La Tène C2 et perdurent à La Tène D1, ce qui rend délicate une attribution chronologique précise. Nous les attribuons donc d'une manière générale à La Tène C2-D. La partie majoritaire du corpus, correspondant aux nombreux bracelets à tige simple, à section triangulaire ou en $\mathrm{D}$, en verre pourpre ou bleu cobalt appartient clairement à La Tène D1.

La nature votive de ce dépôt n'est pas douteuse, en raison des informations dont on dispose sur son contexte de découverte et sur les objets associés. Cet ensemble est unique en son genre; dans l'état actuel de nos connaissances, il ne présente pas d'équivalent en Gaule. La parure en verre celtique n'est pas totalement absente des sanctuaires et il est possible de la retrouver sous forme d'offrandes ou anathemata sur certains sites cultuels, par exemple 
Bennecourt dans les Yvelines (Bourgeois dir., 1999, p. $126-$ 127 et fig. 95) et Mirebeau-sur-Bèze en Côte-d'Or (Bride, 1999). Mais un tel rassemblement, dans une excavation aménagée à cette fin, est inconnu pour cette catégorie de mobilier. Au sein de cet ensemble, de datation assez large, la diversité de teintes, de formes et de combinaisons formecouleur-décor des objets peut être mise en parallèle avec la coexistence de quelques types communs représentés en très nombreux exemplaires et de quelques types rares, voire inédits, aux couleurs inusitées, illustrés en très petit nombre. Le caractère répétitif et l'homogénéité de certaines séries font inévitablement penser à un stock d'artisans verriers. La présence de ces séries monotones, relevant d'une même "patte" (les perles grêles jaune pâle, par exemple), invite à s'interroger sur le statut de ces objets et sur la possibilité qu'ils constituent une production spécifique, au même titre que certaines séries de vases miniatures céramiques, particulières aux sanctuaires et inconnues sur les habitats (Barral, 1994) ; mais on se heurte là à notre médiocre connaissance de l'artisanat verrier gaulois.

A.-S. B.

\section{L'ÉQUIPEMENT DU GUERRIER}

Le nombre de pièces de fourniment métallique d'époque laténienne provenant du sanctuaire de Mandeure n'a rien à voir avec les quantités d'objets de cette catégorie mises au jour sur les sites emblématiques de Gournay-sur-Aronde dans l'Oise (Brunaux et al., 1985 ; Brunaux, Rapin, 1988 ; Lejars, 1994) et de Ribemont-sur-Ancre dans la Somme (Brunaux dir., 1999). Contrairement à ces sanctuaires du nord de la Gaule qui livrent des centaines de pièces d'équipement guerrier, nous n'en dénombrons que vingt-trois à Mandeure (fig. 14).

\section{Description du corpus}

Cet ensemble se compose d'une extrémité proximale d'épée dans son fourreau (fig. 14, $n^{\circ} 1$ ), de quatre fers (fig. 14, $\mathrm{n}^{\text {os }} 2-5$ ) et de trois talons (fig. 14, $\mathrm{n}^{\text {os }} 6-8$ ) d'armes d'hast, ainsi que de quatorze agrafes de ceinturon (fig. 14, $\mathrm{n}^{\text {os } 9-23) \text {. }}$

Le fourreau possède un système d'entrée et de suspension qui permet de le rapprocher de modèles à bouterolle longue de type échelle, rencontrés par exemple dans la vallée de la Saône (Guillaumet, Szabó, 2002). Ces comparaisons permettent d'attribuer le fourreau et l'épée de Mandeure à La Tène C2 et D1.
Parmi les fers d'armes d'hast, on dénombre un fer de javelot (fig. 14, $\mathrm{n}^{\mathrm{o}}$ 2) de 17,6 cm de long, la douille mesurant $7,9 \mathrm{~cm}$; la section de cet objet est losangique; sa morphologie le rapproche du javelot $\mathrm{n}^{\mathrm{o}} 1715$ de Gournay-sur-Aronde (Brunaux, Rapin, 1988, pl. XLVI et p. 128). L'analyse qu'en fait A. Rapin permet d'attribuer ce type de fer à La Tène C2-D1.

Les trois autres fers d'armes d'hast sont des fers de javeline (fig. 14, nos 3-5). Il s'agit d'armes de jet légères, dont la longueur n'excède guère les $10 \mathrm{~cm}$ et dont la chronologie est difficile à établir par leurs seuls caractères typologiques. De telles armes sont souvent attribuées aux phases finales de La Tène. Toutefois, des études récentes menées sur le temple de Mercure au sommet du puy de Dôme permettent d'affirmer qu'elles peuvent encore intégrer les pratiques rituelles jusqu'au début du $\mathrm{II}^{\mathrm{e}} \mathrm{s}$. de notre ère (D. Tardy, J.-L. Paillet, J.-P. Guillaumet, P. Méniel et G. Bataille, étude en cours).

Les trois talons d'armes d'hast (fig. 14, $\mathrm{n}^{\text {os }} 6-8$ ) ont des dimensions qui ne permettent pas de les associer aux différents fers décrits ci-dessus. Ils constituent ainsi un indice de la présence sur ce site d'autres armes d'hast que des armes de jet légères au sein de ce sanctuaire.

Parmi les agrafes de ceinturon, dix sont en fer et cinq en alliage cuivreux. Du point de vue typologique, les objets $\mathrm{n}^{\text {os }} 9,10,11$ et 12 et 13 , du type $4 \mathrm{C}$, sont des modèles de La Tène C2; le $\mathrm{n}^{\mathrm{o}} 14$, du type $4 \mathrm{~A} 2$, et les $\mathrm{n}^{\text {os }} 14$ et 15 , du type 4B (à languette en V), sont des agrafes de La Tène $\mathrm{D} 1$; les $\mathrm{n}^{\mathrm{os}} 17,18,19,20$ et 21 , également de type 4B, mais à languette atrophiée, ont une perduration plus longue, de tels modèles se retrouvant encore à La Tène D2 ; enfin, les $n^{\text {os }} 22$ et 23 sont des agrafes du type 4E2, à excroissances latérales atrophiées. Il s'agit d'objets dont les premiers exemplaires apparaissent à La Tène D1, mais qui sont surtout caractéristiques de La Tène D2 et peuvent encore se retrouver dans des contextes augustéens (Bataille, 2001).

\section{Analyse et synthèse}

À la vue de ce corpus, on constate que les pratiques rituelles du sanctuaire de Mandeure n'assimilent qu'un petit nombre de pièces de l'équipement guerrier (si les trouvailles qui nous sont parvenues reflètent bien la réalité des dépositions, ce qui est loin d'être certain, vu les conditions de découverte). Si l'armement peut paraître anecdotique comparé aux autres mobiliers et surtout à l'impressionnante collection d'objets en verre celtiques, il n'en est pas moins intéressant dans la mesure où il témoigne d'une relative diversité des mobiliers soumis aux pratiques d'offrande ou de sacrifice au sein de ce sanctuaire. 

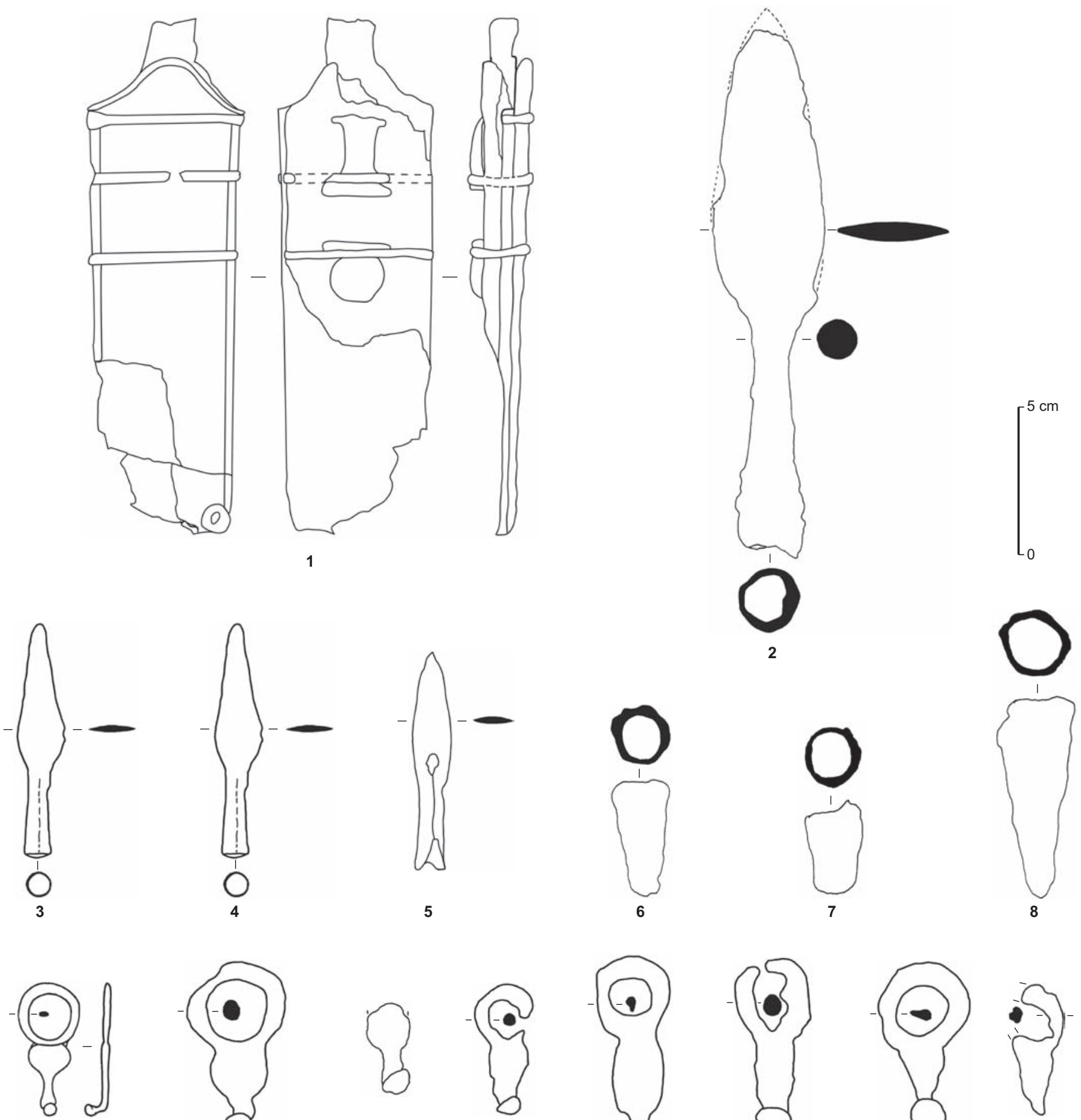

9
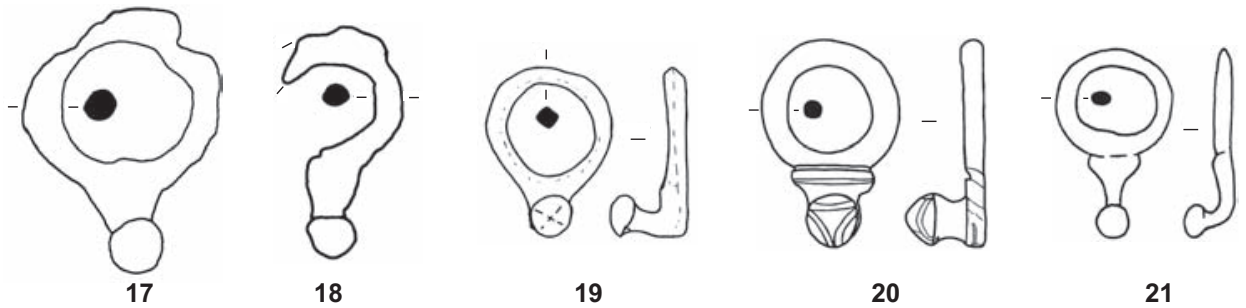

21

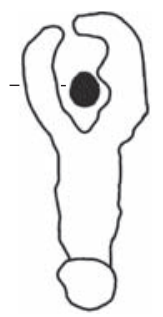

14

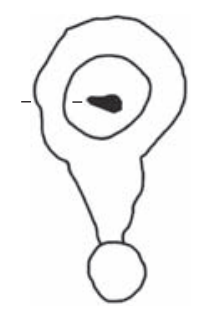

15

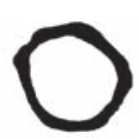

Fig. 14 - Le Clos du Château à Mandeure. L'armement provenant du sanctuaire : 1, fourreau et épée ; 2, fer de javelot ; 3-5, fers de javeline ; 6-8, talons d'armes d'hast ; 9-23, agrafes de ceinturon (1-19, fer; 20-23, base cuivre) (dessins : $n^{\circ} 1$, G. Bataille ; $n^{\text {os }} 3-18,21-23$, R. Pierson; $n^{o s}$ 19-20, V. Pichot; DAO : G. Bataille). 
La chronologie des artéfacts est également très intéressante. De ce point de vue, la comparaison n'est pas pertinente avec Gournay-sur-Aronde, l'armement de ce site disparaissant à la fin de La Tène C2 ou au tout début de La Tène D1, soit au moment où les premières armes apparaissent à Mandeure. Sur ce sanctuaire séquane, les plus anciens éléments sont l'épée dans son fourreau et les quatre agrafes de ceinturon qui datent de La Tène C2. Aux phases suivantes (La Tène D1 et D2), seules des agrafes de ceinturon et des armes de jet intègrent le mobilier du sanctuaire. Il a été démontré récemment qu'à partir de la fin de La Tène C2, les agrafes de ceinturon deviennent les objets de la panoplie guerrière les plus courants sur les sanctuaires et qu'à partir de La Tène D1 apparaissent des armes de jet légères, comme celles de Mandeure (Bataille, 2004). Ce sanctuaire, qui paraît atypique par sa faible quantité d'armes, ne l'est finalement pas dans la représentation chronologique de cette catégorie de mobilier. Les pièces de fourniment des deux derniers siècles de La Tène sont certes minoritaires à Mandeure, mais leur assemblage rappelle celui d'autres lieux de culte, comme La Villeneuveau-Châtelot dans l'Aube (Bataille, 2003 et 2004), site qui livre pourtant une majorité d'objets de fourniment, quelle que soit la période envisagée.

Les pratiques intégrant des équipements guerriers ne semblent donc pas prééminentes à Mandeure, comme l'attestent les vestiges qui en découlent et qui nous sont parvenus. Néanmoins, l’image qui en résulte est cohérente avec les observations réalisées sur d'autres lieux de culte à cette même époque. Par conséquent, Mandeure s'intègre parfaitement dans le paysage des sanctuaires celtiques, malgré ces spécificités bien affirmées.

G. Ba.

\section{FEUILLES, CARNYX ET ENSEIGNES}

On dispose de peu d'informations sur les conditions de découverte de ces objets (voir infra, p. 390). C. Duvernoy énumère pêle-mêle : «[...] des lames de bronze estampées ou repoussées représentant de grossières figures d'animaux, une feuille de lierre bien conservée, quelques débris d'armes [...]»(Duvernoy, 1883b, p. 42-43).

\section{Éléments foliacés}

Les cinq feuilles ou éléments foliacés déposés aux musées de Montbéliard et de Besançon, font partie des objets découverts lors des fouilles du sanctuaire à la fin du
$\mathrm{XIX}^{\mathrm{e}}$ s. et au début du XX $\mathrm{X}^{\mathrm{e}}$ s. (fig. 15). Seule celle déposée au musée de Besançon (5890.2.2) a fait l'objet d'un article par W. Kimmig en 1977 (fig. 15, no 1).

En tôle d'alliage base cuivre, elles sont toutes fabriquées suivant la même technique. La nervure creuse est créée par emboutissage sur une forme, ainsi que la moitié de l'embout qui lui fait suite. La transformation en tige creuse est réalisée pour la deuxième partie par repli de tôle réservée à la base de la feuille lors de sa découpe. Les bords sinueux aux courbures marquées sont découpés suivant un patron en forme de poire. Toutes les nervures, sauf celle du $n^{\circ} 4$, se terminent dans la pointe. Lorsque l'on observe les feuilles posées à plat nervure vers le haut, quatre ont la nervure tournée vers la droite et une, la $n^{0} 1$, vers la gauche. Les $\mathrm{n}^{\text {os }} 1$ et 2 forment une paire exactement semblable, mais en symétrie inversée. Sur le revers des pièces $n^{\text {os }} 1,2$ et 4 , nous observons dans le premier quart de la feuille une corrosion différente, due au contact avec un autre objet assez large, au bord légèrement sinueux, peut-être la trace d'une autre feuille.

Ces feuilles sont connues depuis le $\mathrm{XIX}^{\mathrm{e}} \mathrm{s}$. sur des sites de la fin de l'époque protohistorique. Le site de Abentheuer, Kr. Birkenfeld en Allemagne, en a livré une paire (Haffner, 1976, p. 171-172, pl. 129). Les sites de Sanzeno, province de Trento en Italie (Oberosler, in Collectif, 2004, p. 680), et de La Tène à Marin, canton de Neuchâtel en Suisse (Vouga, 1923, p. 63, pl. 19, no 1 ), en possèdent chacun un exemplaire. Récemment la découverte de Tintignac, commune de Naves (Corrèze), nous permet d'ajouter à ce très petit corpus au moins quatre exemplaires de très grand taille, d'après les photographies publiées (Lontcho, 2005 ; Maniquet, 2005a et b) ${ }^{8}$.

Les feuilles de Mandeure, avec une taille entre $11 \mathrm{~cm}$ et $15 \mathrm{~cm}$ sans la tige, sont les plus petites connues. Les exemplaires d'Abentheuer, de La Tène et sans doute de Tintignac sont au moins deux fois plus grandes.

La signification et l'usage de ces feuilles restent mystérieux. D'après l'orientation de leurs douilles, il faudrait d'ailleurs plutôt les appeler flammes, en fonction de la typologie proposée par W. Kimmig. P. Vouga y voyait des décors de casques (Vouga, 1923, p. 63); bien d'autres possibilités ont été avancées, elles ont toutes été réfutées

8. Un éclairage nouveau vient en effet d'être donné à cette catégorie d'objets par la découverte récente, dans le sanctuaire de Tintignac à Naves (Corrèze), d'une fosse renfermant près de 500 éléments métalliques, dont des enseignes et cinq carnyx, démontés, accompagnés de casques et autres objets. Ce dépôt serait datable, pour la majorité des objets, de la fin du $\mathrm{II}^{\mathrm{e}}$ et du $\mathrm{I}^{\mathrm{er}}$ s. av. J.-C., quelques-uns paraissant plus anciens : fin du IV $^{\mathrm{e}}-\mathrm{III}^{\mathrm{e}}$ s. (Maniquet, 2005a et b). 

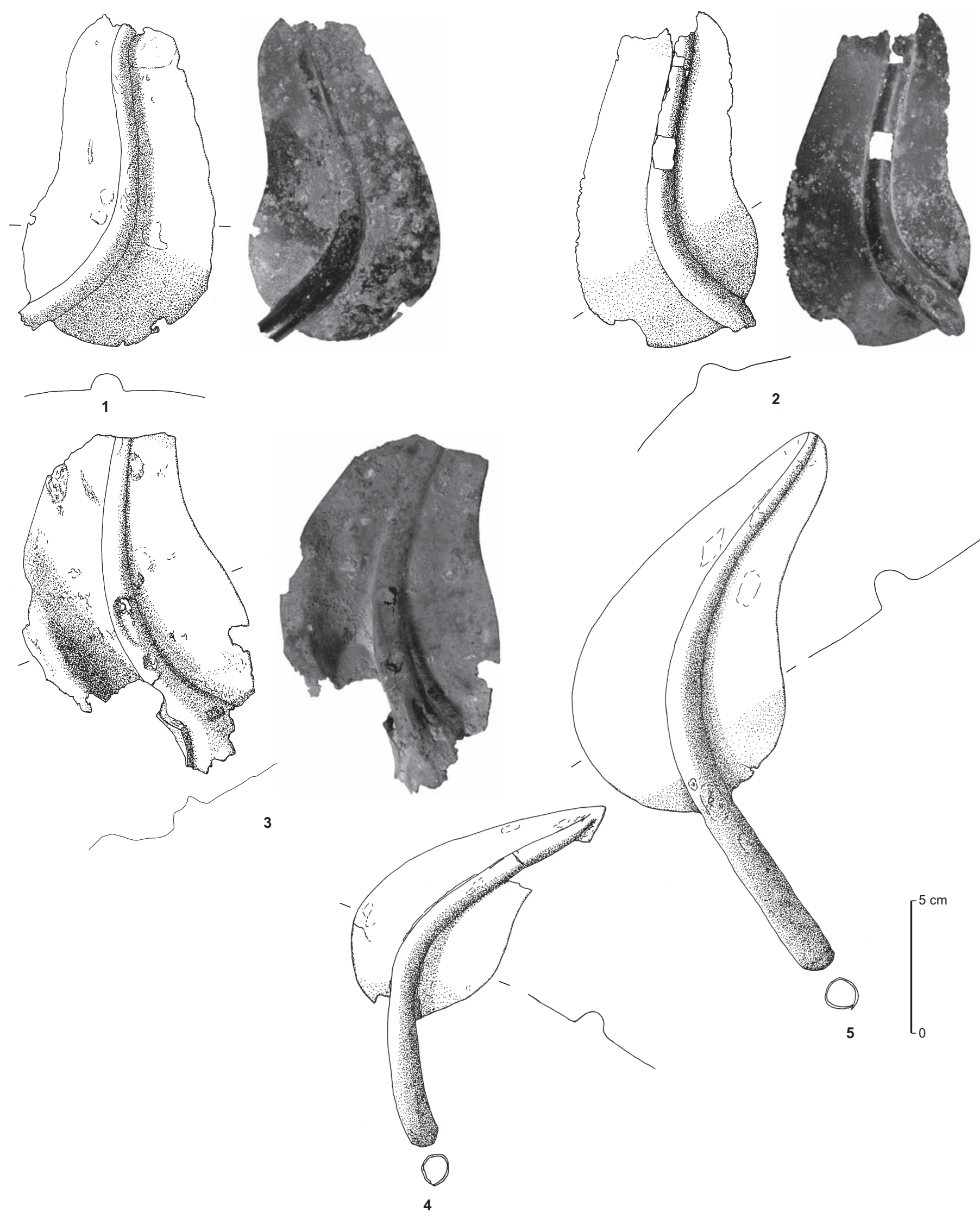

Fig. 15 - Le Clos du Château à Mandeure. Éléments foliacés attribués au sanctuaire gaulois : 1-4, conservés au musée de Montbéliard ; 5, conservé au musée de Besançon (dessins : V. Pichot ; clichés : A. Aubert, musée de Montbéliard ; DAO : P. Barral, LCE). 

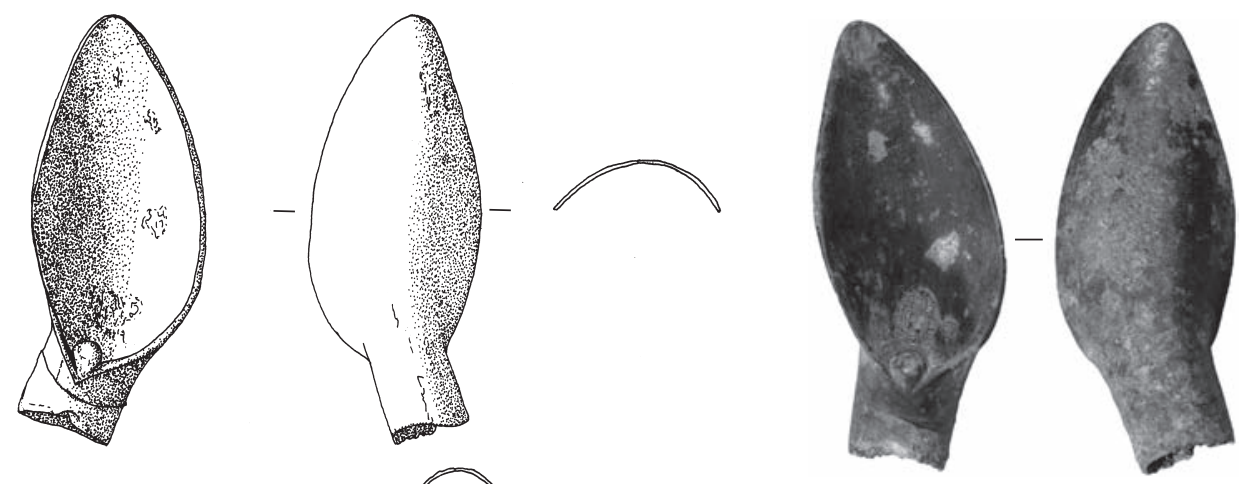

1
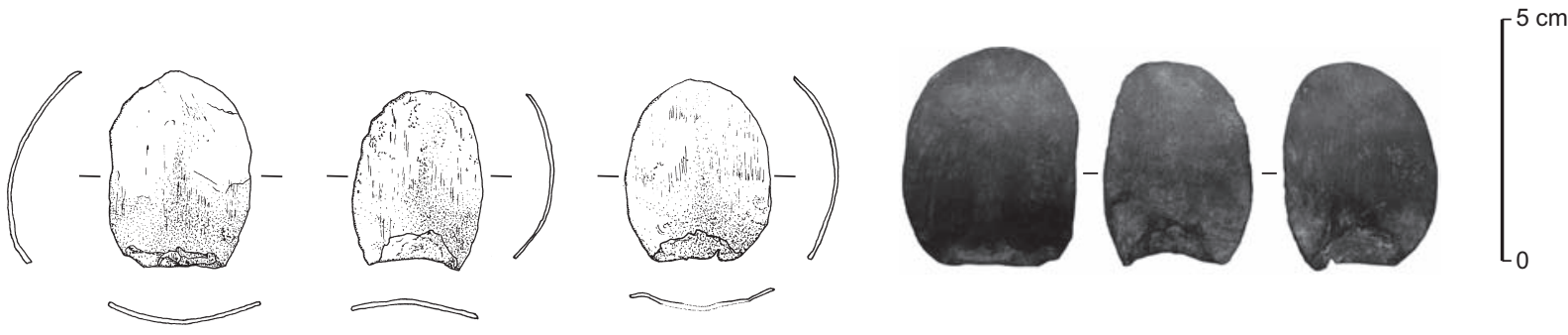

Fig. 16 - Le Clos du Château à Mandeure. Éléments interprétés comme oreilles d'animaux, attribués au sanctuaire gaulois, conservés au musée de Montbéliard (dessins : V. Pichot; clichés : A. Aubert, musée de Montbéliard; DAO : P. Barral, LCE).

par W. Kimmig (1977, p. 79-80). Lors d'une exposition récente à Montbéliard, on a proposé d'en faire les feuilles d'un arbre, dans l'esprit de celui découvert à Manching (Lkr. Pfaffenhofen an der Ilm, Allemagne) (Maier, 2001). C. Maniquet, pour sa part, les considère comme des oreilles de carnyx (Maniquet, 2005a et b). En effet, à la base du dépôt de Tintignac, deux feuilles semblent en connexion à la base de l'embouchure d'un instrument. Les travaux d'études permettront de confirmer cette observation très importante pour l'interprétation de ces feuilles. En effet, toute l'iconographie des carnyx connue d'après des représentations sur des monnaies (Homo-Lechner, Vendries, 1993, p. 33-36) et des bas-reliefs (par exemple l'arc d'Orange: Amy etal., 1962) montrent des carnyx dotés de petites oreilles, dont la taille est sans commune mesure avec celle des feuilles présentées ci-dessus.

\section{Oreilles d'animaux}

Une véritable oreille d'animal, en alliage base cuivre coulé, se trouve au musée de Montbéliard (fig. 16, no 1 ) ; elle fait partie de l'ensemble d'objets attribués au sanctuaire. Par sa forme et par sa taille, elle appartiendrait à un cervidé, représenté grandeur nature. La fixation à la tête de l'animal s'effectuait au moyen d'un tube cylindrique soudé à sa base. Cet élément est comparable aux oreilles trouvées à La Tène (Vouga, 1923, pl. 19, no 2) et du gué de Salcy à Sens dans l'Yonne (Rolley, in Guillaumet et al., 1982 , p. $35, \mathrm{n}^{\circ} 73$ ). Ces oreilles appartiennent probablement à une statue en bois de grande taille sur laquelle étaient ajoutées des parties métalliques. Le cheval de Manching, composé de plusieurs éléments en fer, semble une exception (Krämer, 1998).

Trois oreilles, simples tôles légèrement courbées de forme ovale, portant des traces de soudure à l'étain à la base, sont des témoins d'autres représentations animales (fig. 16, no 2). Elles sont comparables à celles des sangliersenseignes de Neuvy-en-Sullias dans le Loiret et Soulacsur-Mer en Gironde (Moreau et al., 1990 ; Ankner et al., 1995), mais peuvent aussi être rapprochées des représentations d'oreilles de carnyx des monnaies et surtout de l'arc d'Orange. 


\section{Carnyx}

Les trois fragments de carnyx de Mandeure, en tôle d'alliage base cuivre travaillée au repoussé, font partie de deux têtes distinctes (fig. 17). Les différences portent sur la forme générale des têtes de sanglier avec les défenses bien marquées, le rendu des dents, le modelé des joues et des yeux (emplacement et angles). La bouche est grande ouverte. L'œil est ajouté. Il devait être réalisé dans un autre métal plutôt qu'en pâte de verre, en raison des traces grises subsistant qui semblent de la soudure à l'étain et mériteraient une analyse de composition. Une ouverture ovale dans les tôles, à l'emplacement de l'oreille, avec des traces de soudure à l'intérieur, permet d'affirmer que les oreilles sont des éléments ajoutés comme pour les enseignes. Les trois oreilles, simples tôles ovales, mentionnées ci-dessus, pourraient également faire partie de ces représentations.

Avant la découverte de Tintignac, ces fragments étaient les seuls éléments de carnyx (ou carnyces) connus en Gaule. D'autres exemplaires sont connus: à Deskford (Écosse), exemplaire assez tardif avec une tête figurative (Hunter 2001), à Castiglione delle Stiviere, province de Mantoue, dans une tombe richement dotée, avec une tête décorée au repoussé (De Marinis, 1997). Il existe aussi un pendentif miniature en bronze dans la tombe 9 de Bouy, dans la Marne (Homo-Lechner, Vendries, 1993, p. 28-37 ; Collectif, 2002, p. 103-111), une iconographie importante sur les monnaies celtiques et romaines, et l'arc d'Orange. Une partie courbée tubulaire, sans le pavillon, est connue dans le dépôt $\mathrm{D}$ en milieu humide de Dürnau-Vollocher Ried à Kappel, Kreis Biberach, Allemagne (Wieland, 1996, p. 107, pl. 5B1).

\section{Enseigne}

Deux fragments de crête à décor ajouré et un groin en tôle en base cuivre sont attribuables à un ou deux sangliersenseignes du même genre que ceux de Soulac-sur-Mer en Gironde (fig. 18). Le fragment de crête principal se présente comme un élément de tôle circulaire, lié aux départs de deux rinceaux (fig. 18, $n^{\circ} 1$ ). D'autres rinceaux devaient se développer sur le pourtour de l'élément circulaire, comme le suggèrent d'infimes traces de découpe. Cet élément circulaire porte deux cercles concentriques gravés sur sa bordure et présente dans sa partie centrale deux échancrures en forme de feuilles ou virgules opposées et inversées; on peut le rapprocher de disques provenant de Hongrie (Schönfelder, 2002, p. 263, fig. 164, $\mathrm{n}^{\text {os }} 6$-7). Un autre petit fragment rappelle assez précisément certains motifs de la crête du sanglier de Soulac-sur-Mer (fig. 18, $n^{\circ}$ 2).

Un troisième fragment de tôle, à décor gravé et obtenu au repoussé, présentant un pourtour déchiqueté, correspond selon nous à un groin avec deux naseaux fortement marqués en creux, extrémité sertie maintenant des deux parties de la coque figurant la hure d'un sanglier (fig. 18, no 3).

Un dernier fragment de tôle en alliage base cuivre travaillé au repoussé présente des affinités avec les fragments de carnyx décrits ci-dessus, mais s'en distingue par le détail du décor et la patine du bronze (fig. 18, no 4). Ce fragment, qui a été très malmené, présente la trace nette d'un oil et des motifs décoratifs sinueux en relief. Nous verrions plus volontiers dans cet élément un fragment de hure de sanglier-enseigne que de carnyx.

Les enseignes en forme de sanglier suivent les mêmes règles de déposition que les feuilles et les carnyx. Les exemples archéologiques sont rares : Soulac-sur-Mer, Neuvy-en-Sullias, Ilonse (Alpes-Maritimes) (Lavergne, 2000) et Tintignac. En revanche, les représentations sont abondantes.

\section{Conclusion}

Ces carnyx, feuilles et enseignes de Mathay-Mandeure font partie d'un dépôt de sanctuaire semblable à ceux de Neuvy-en-Sullias et Tintignac. La découverte d'éléments très fragmentés lors des recherches du XIX ${ }^{\mathrm{e}} \mathrm{s}$. et du début $\mathrm{du} \mathrm{XX}^{\mathrm{e}} \mathrm{s}$. nous incite à penser que le dépôt primaire avait déjà été bouleversé et dispersé lors de la construction du sanctuaire romain monumental. Ces pièces témoignent cependant de pratiques encore mal connues de déposition d'objets liés à la guerre dans des enceintes religieuses. Si les enseignes et les carnyx constituent un thème récurrent dans l'iconographie romaine pour consacrer la défaite des armées gauloises, au même titre que les panoplies guerrières, on ne dispose en revanche d'aucune représentation de feuilles ou flammes. Peut-être leur signification était-elle aussi mystérieuse pour les Romains que pour nous.

J.-P. G., M. S.

\section{CONCLUSION SUR LE SANGTUAIRE GAULOIS}

Le caractère composite des trouvailles du sanctuaire de Mandeure rapproche nettement ce site de celui de Mirebeau-sur-Bèze en Côte-d'Or (Barral, Guillaumet, 1994 ; Joly, Barral, à paraître), et distingue clairement ces deux derniers, du point de vue des assemblages mobiliers, des sanctuaires du nord de la Gaule. 


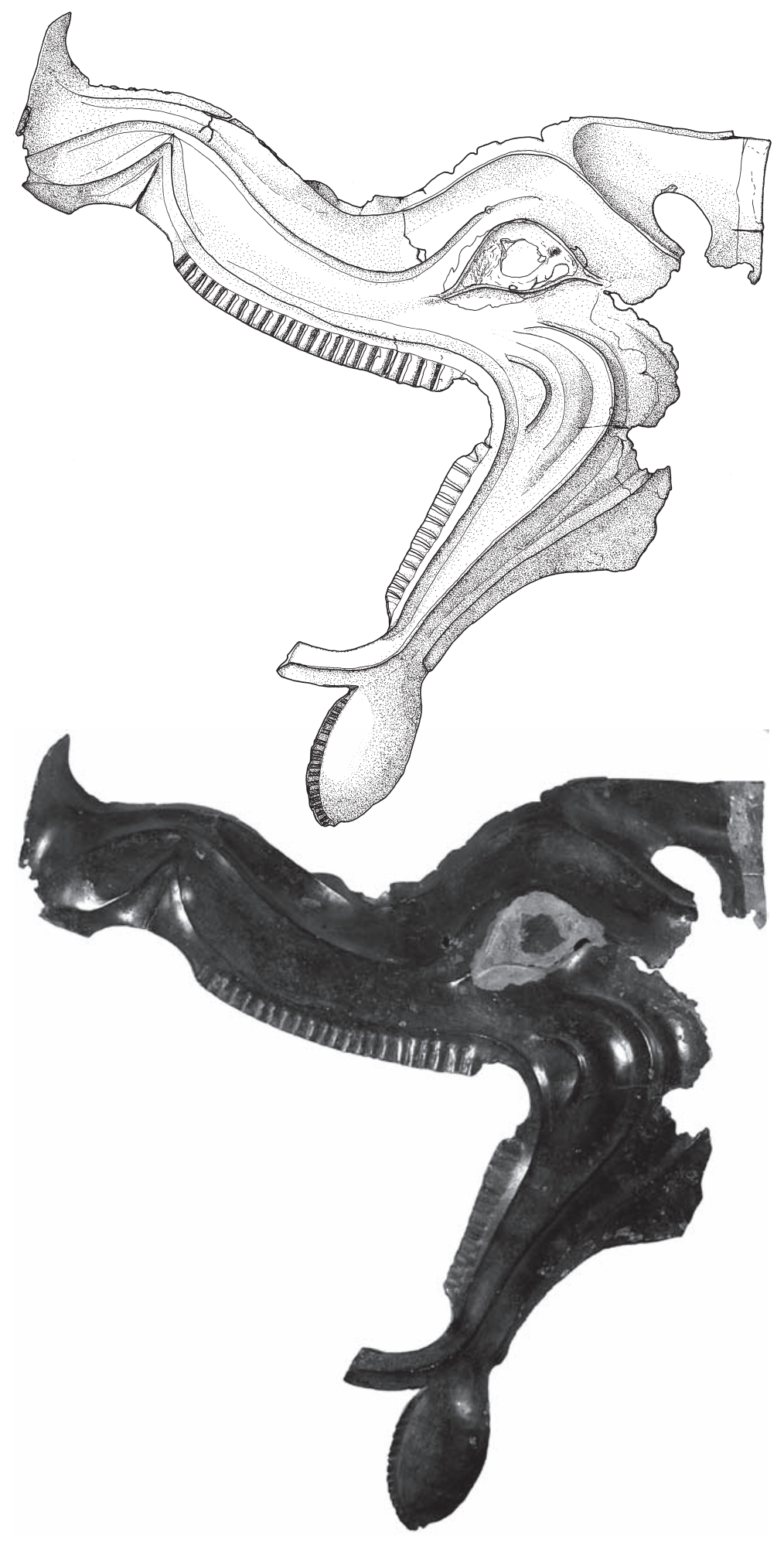

1
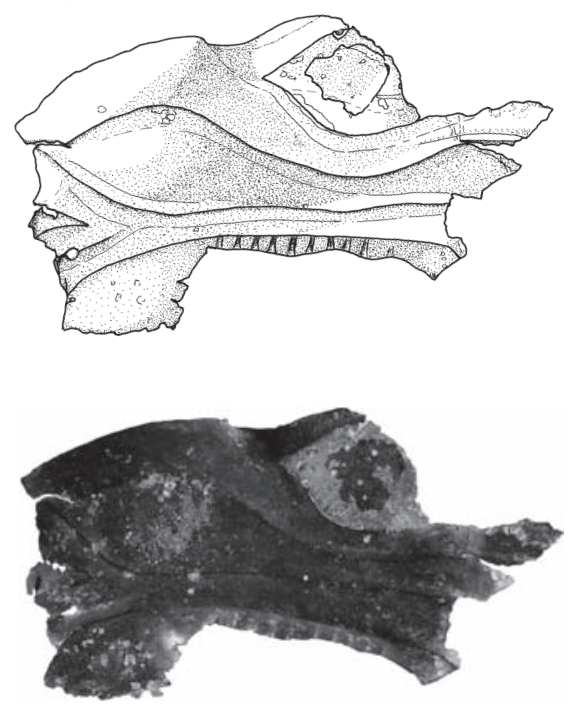

2
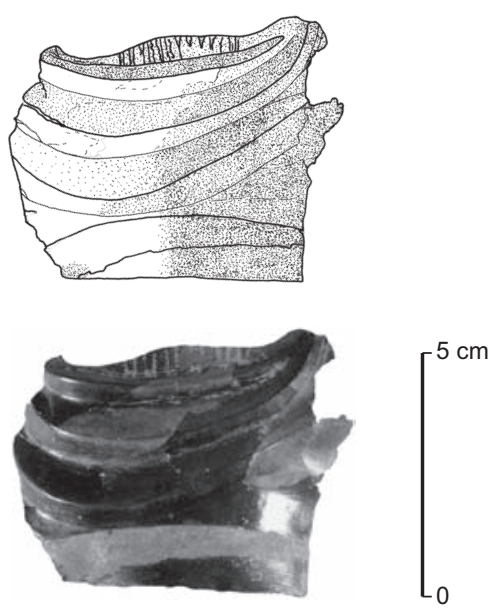

3

Fig. 17 - Le Clos du Château à Mandeure. Fragments de pavillons de carnyx attribués au sanctuaire gaulois, conservés au musée de Montbéliard (dessins : V. Pichot; clichés : A. Aubert, musée de Montbéliard ; DAO : P. Barral, LCE). 


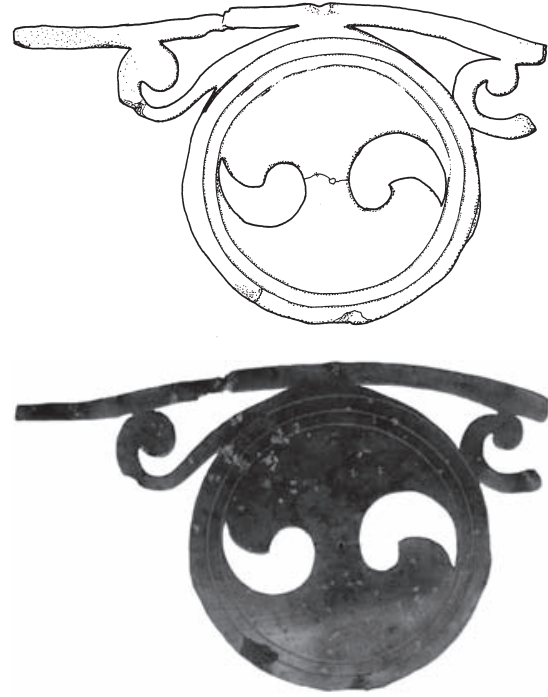

1

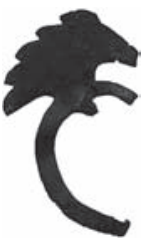

2

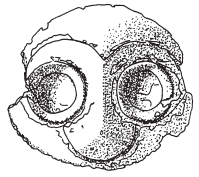

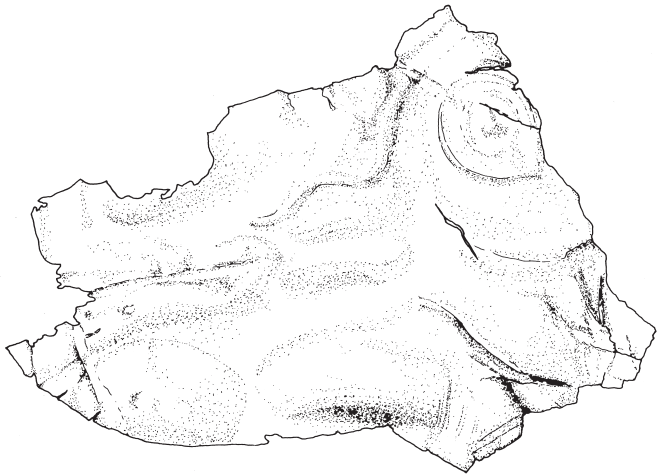

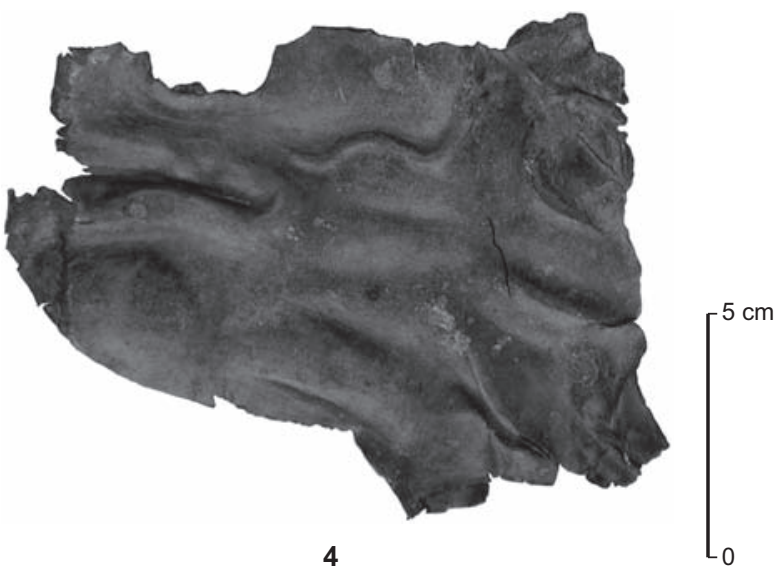

4

Fig. 18 - Le Clos du Château à Mandeure. Fragments interprétés comme parties d'enseignes, attribués au sanctuaire gaulois, conservés au musée de Montbéliard (dessins: V. Pichot; clichés : A. Aubert, musée de Montbéliard; DAO : P. Barral, LCE).

L'ensemble principal (découverte Péquignet) est emblématique d'un type de dépôt dans lequel une série majoritaire d'objets peu fragmentés et présentant une indéniable homogénéité de fabrication et de datation (La Tène C2-D1) se trouve associée à des éléments de types variés, fragmentaires, mutilés, parfois contemporains du lot principal, parfois plus anciens ou au contraire plus récents que ce dernier, illustrant les modalités complexes de présentation, déposition, enfouissement des objets dans les sanctuaires.

La série d'objets de parure en verre soulève évidemment une interrogation. À titre de comparaison, le sanctuaire de Mirebeau-sur-Bèze, fouillé quasi exhaustivement, n'a livré pour la même période qu'une trentaine de perles et bracelets en verre, répartis entre de nombreux contextes. Le rassemblement et l'enfouissement d'un grand nombre d'objets de même catégorie illustrent un geste votif collectif bien attesté au second âge du Fer (voir par exemple la trouvaille de Duchcov). Le choix de la matière première et de la catégorie fonctionnelle concernée ici revêt sans doute une signification particulière. Les perles et anneaux en verre coloré, de même que d'autres séries bien attestées à Mandeure, quoique en plus faible nombre (fibules, anneaux en bronze ou en lignite...), présentant une unité de réalisation certaine, renvoient à l'existence d'artisanats spécialisés dans la fabrication en série de petits objets. Est-il dans ces conditions exagéré d'évoquer l'offrande d'une communauté d'artisans verriers locaux, ou la commande d'un puissant membre de l'aristocratie séquane ayant la mainmise sur cet artisanat?

Carnyx et enseignes, volontairement démantelés et enfouis, pourraient constituer des trophées pris à l'adversaire et offerts aux divinités guerrières, dans un contexte de conflits entre tribus. Il peut s'agir également de pièces désacralisées, car arrivées en fin d'utilisation ou devenues désuètes, mais dont la forte charge symbolique et religieuse nécessitait, pour les réformer, des manipulations rituelles particulières. À ce jour, Mandeure et Tintignac sont les seuls sites de sanctuaire où se trouvent associées trompes de guerre et enseignes. Les affinités stylistiques entre les pavillons de carnyx de Mandeure et certains exemplaires de 
Tintignac suggèrent, sinon une fabrication par un même atelier, au moins l'existence d'un même modèle.

L'importance du sanctuaire gaulois de Mandeure peut se déduire du nombre et de la qualité des objets découverts au XIX ${ }^{\mathrm{e}}$ s., mais aussi de la construction, au Haut-Empire d'un complexe monumental associant théâtre (l'un des plus grands de Gaule par ses dimensions), temples et probablement thermes. La ville florissante du Haut-Empire se trouve ainsi au bout d'un processus initié avec la naissance d'un grand sanctuaire de territoire, vers la fin $d u I^{e} s$. ou le début du III $^{\mathrm{e}}$ s. av. J.-C. L'emplacement du sanctuaire de Mandeure, à la croisée de deux axes majeurs de communication, dans une zone de transition entre les territoires séquane et rauraque (Fichtl, 2000 ; Jud, Kaenel, 2002), était propre à favoriser l'émergence d'un pôle de peuplement et une dynamique proto-urbaine.

P. B.

\section{LE SANCTUAIRE GALLO-ROMAIN}

\section{HISTORIQUE DES RECHERCHES}

Les premières trouvailles sur le site du Clos du Château remontent au XVIII ${ }^{\mathrm{e}}$ s. L. Parrot effectue, en effet, quelques recherches sur ce lieu, entre 1783 et 1785 . Les résultats sont rapportés dans une note manuscrite du 23 mars 1785 et une conférence du 20 avril 1785 à l'Académie de Besançon, et ses travaux seront «pillés» par C. Duvernoy (Jeannin, 1986, p. 43). Au XIX ${ }^{\mathrm{e}}$ s., F. Morel-Macler signale la découverte, en 1837, de fragments d'architecture trouvés «dans les derniers vestiges au midi du Doubs, sur la rive droite » (Morel-Macler, 1847, pl. 4 et 5). À cet endroit, s'élevaient encore à cette époque des vestiges imposants, sous la forme de trois énormes murgers. La récupération de matériaux pour la construction est à l'origine d'une série de découvertes fortuites, en 1880-1881, qui attirèrent l'attention de deux notables et érudits locaux, C. Duvernoy et H. L'Épée (Castan, 1882, p. 264 ; Jeannin, 1986). Ceux-ci vont entreprendre les premières fouilles "scientifiques", en 1882 et 1883 ; ils tentent de «canaliser» les actions des paysans locaux, de faire dégager l'ensemble des vestiges et d'en établir le plan. Ces recherches eurent lieu dans un climat difficile, chaque propriétaire d'une des nombreuses parcelles concernées cherchant à tirer un bénéfice des objets mis au jour en les vendant au plus offrant. En 1883, C. Duvernoy présente les découvertes à la Société des Antiquaires de France et à la Sorbonne (27 juin 1887), exposés qui donnent lieu à un article publié à la fois dans les Mémoires de la Société d'émulation de Montbéliard de 1882 (Duvernoy, 1883a) et dans le Bulletin de la Société des Antiquaires de France (Duvernoy, 1883b). L'édifice est alors interprété comme une basilique gallo-romaine, située à l'intérieur de l'enceinte d'un marché aux chevaux, en raison de la grande quantité de monnaies et de clochettes mises au jour à cet emplacement.

En fait, les recherches de «trésors » sur les différentes parcelles du site ont continué jusqu'en 1914 et les objets recueillis furent dispersés, au gré des ventes, dans les collections de plusieurs musées (dont ceux de Besançon, Montbéliard, Belfort, Mulhouse, Bâle, Saint-Germain-enLaye).

Enfin, en 1933, H. Koethe identifie les vestiges comme les restes d'un sanctuaire gallo-romain (Koethe, 1933). En 1986, Y. Jeannin décrit l'édifice, désigné sous le terme de «temple gaulois", comme un «temple à base rectangulaire [...]»(Jeannin, 1986, p. 53). Les publications postérieures se contentent de paraphraser cette description.

La documentation graphique disponible est constituée par le plan des fouilles de 1882, effectué le 27 juin 1883. Ce dernier a été publié par C. Duvernoy (1883a et b) (fig. 19). Cet auteur présente dans ces deux articles un compte rendu des fouilles réalisées, ainsi qu'un inventaire succinct des découvertes les plus spectaculaires. En outre, les archives du musée conservent un document manuscrit, dit plan Barbier, établi en 1893 (Jeannin, 1986, p. 44, p. 47, p. 57, note 6 et fig. 15, p. 65). Sur ce plan en quatre feuilles, à l'échelle $1 / 1250$, prenant pour fond le cadastre de 1845, sont localisés le théâtre et le sanctuaire, ainsi que les murgers relevés par F. Morel-Macler en 1824 (fig. 3). Quelques murs inexistants sur le plan de 1882 y figurent. L'ensemble a été réuni et analysé par Y. Jeannin, vers le milieu des années 1980 (Jeannin, 1986). Par la suite, les informations ont été reprises dans diverses publications consacrées à Mandeure et qui évoquent le sanctuaire (voir en particulier : Frézouls et al., 1988). Il semble cependant utile de reprendre la documentation afin de tenter d'interpréter objectivement les découvertes du XIX ${ }^{\mathrm{e}} \mathrm{s}$. Les plans conservés montrent un ensemble qui comporte une enceinte ovale, à l'intérieur de laquelle prend place une construction de plan quadrangulaire.

\section{L'ENCEINTE}

L'enceinte présente une forme ovale, d'un diamètre de 117,80 m (sens est-ouest) par 110,70 m, et dessine un polygone à 36 côtés; elle couvre une surface de plus de 